\title{
Estructuras de comunicación en el campo de la ciencia social en Chile: un Análisis de Redes ${ }^{1}$
}

\author{
Claudio Ramos Zincke²- Universidad Alberto Hurtado, Chile
}

\begin{abstract}
Resumen
El objetivo de este artículo es analizar las redes existentes en el campo de la ciencia social en Chile y la incidencia de diversos factores sociocognitivos sobre la configuración estructural de tales redes. Para ello, analizo publicaciones que reportan resultados de investigación, tomando como base empírica las redes de citas y las redes de coautoría. Tal estudio lo hago con respecto a tres disciplinas: sociología, ciencia política y antropología, para el período 2000-2006, analizando 21.787 referencias.

Los resultados muestran que las redes de comunicación científico social en el país son poco densas, con muy baja interdependencia y reciprocidad, baja conglomeración, y forma predominantemente jerárquica. En contradicción a la generalizada valoración de la interdisciplinariedad, se constata que las disciplinas ponen fuertes barreras a la comunicación. Los encuadramientos paradigmáticos, por su parte, aunque son fuertes atractores de conglomeración, son mucho menos excluyentes y existe un grupo reducido de investigadores que operan como mediadores interparadigmáticos. La investigación reveló además un significativo rol del género en la constitución de las redes. El análisis de redes permite, así, develar un conjunto de pautas de estructuración del campo de las ciencias sociales y contribuye a comprender su forma de operación.
\end{abstract}

Palabras clave: Redes sociales, paradigma científico, campo científico, ciencia social

\begin{abstract}
The purpose of this article is to analyze the existing networks in the field of social science in Chile and the incidence of diverse sociocognitive factors on the structural configuration of such networks. With that aim, I study publications that report research results, taking as empirical basis the network established through citations and the networks of coautorship. I made such study with respect to three disciplines: sociology, political science and anthropology, for the period 2000-2006, analyzing 21,787 references.

The results show that the social scientific communications networks in the country are scarcely dense, with very low interdependence and reciprocity, and with low conglomeration, assuming a predominantly hierarchic form. In contradiction with the generalized valuation of interdisciplinarity, it is acknowledged that disciplines put strong barriers to the communication. The paradigmatic frameworks, on the other hand, although are strong atractors of conglomeration, they are much less excluding

\footnotetext{
${ }^{1}$ Este texto es producto de una investigación con financiamiento de Fondecyt (Proyecto $N^{\circ} 1070814$ ). Su realización fue facilitada por una estadía del autor como visiting scholar, durante el 2010, en la University of Texas at Austin, que contó con el apoyo del Programa MECE Educación Superior y de la Universidad Alberto Hurtado.

2 Email: cramos@uc.cl y claudior@uahurtado.cl
} 
and exists a reduced group of investigators who operate like interparadigmatics brokers. The investigation revealed in addition a significant role of gender in the constitution of networks. The analysis of networks allows, thus, to reveal a set of patterns structuring the field of social sciences and contributes to understand its form of operation.

Key words: Social networks, scientific paradigm, scientific field, social science

\section{Introducción. Comunicación científica y redes}

\section{Sistema de comunicación, campo científico y su interconexión por la vía de las citas}

El sistema de las comunicaciones científicas expresado en las publicaciones académicas y el campo de las relaciones entre los científicos, que incluye las estrategias de posicionamiento de sus integrantes, son dos dimensiones del proceso científico, que se condicionan recíprocamente (Ramos, 2012). Las estrategias de los investigadores en posiciones periféricas, en su búsqueda de crédito científico - de capital científico, como dice Bourdieu (2001)- les harán, eventualmente, apelar a enfoques teóricos alternativos, innovativos, y buscar aliados que les permitan sumar fuerzas, todo lo cual se manifestará en las publicaciones mismas, que contendrán tales planteamientos teóricos y los nombres de los autores que se busca enrolar. Para la defensa de las propias posturas, además, se valdrán de diversos artificios retóricos que acentúen la fuerzas de los propios argumentos y minimicen sus debilidades (Latour, 1986; KnorrCetina, 2005). Investigadores con elevado reconocimiento académico en el campo tendrán fácil acceso a publicar libros en editoriales prestigiosas, mientras que otros autores, en posiciones periféricas sólo podrán acceder a editoriales emergentes, de mercados estrechos. Así, diferentes características del campo científico y de las posiciones que ocupan los investigadores en él repercuten en lo que ocurre en las publicaciones científicas, dejan su marca en las comunicaciones resultantes.

Por su parte, el conjunto de las publicaciones, que a cada momento se multiplica, provee a los investigadores su material cognitivo de trabajo. De su revisión y selección extraerán los elementos para un nuevo ciclo de producción de conocimientos y generación de nuevas publicaciones. Pero, en las publicaciones -y en los sistemas de indexación de creciente uso- encuentran, junto a las menciones explícitas que hacen unos académicos a otros, señales y pistas con respecto al reconocimiento que se otorga a determinados autores, al prestigio especial logrado por algunos, a las alianzas o ataques que se producen entre autores y corrientes científicas. De ello, los investigadores, con mayor o menor claridad, con mayor o menor conciencia, infieren 
las estrategias que están en juego, infieren la situación en el campo científico o, al menos, en la zona de él que les interesa; e infieren conexiones extracientíficas que vinculan la labor científica con estrategias políticas de influencia. Les ayudan para todo eso, ciertamente, los conocimientos más directos que tienen sobre los actores e instituciones que pueblan los campos científicos locales. Los reconocimientos que hagan en el contenido de las publicaciones y las inferencias que establezcan a partir de ellos les servirán a los investigadores para orientar sus acciones y estrategias en el campo: los llevarán eventualmente a invitar, a un seminario o a otra actividad académica, a determinados autores, que en muchos casos no eran del círculo de conocimiento cotidiano, para establecer vínculos de colaboración. Es decir, así como las dinámicas del campo marcan las publicaciones y dejan huellas en ellas, el contenido de las publicaciones, incluyendo esas huellas, incide a su vez en el campo.

En la conexión entre esta doble dinámica, en el engranaje de esta doble dimensionalidad de la ciencia, un elemento que juega un rol crucial es la cita bibliográfica, la referencia explícita que se hace a la obra de otro autor.

El sistema moderno de referencias, que especifica el texto citado, con su autor y fecha de publicación, cumpliendo una función de enlace argumentativo y de apoyo empírico con otros textos, es de fines del siglo XIX (Leydesdorf \& Wouters, 1999), y viene asociado al desarrollo de la reflexividad de la ciencia y del perfeccionamiento de su proceso de observación de segundo orden.

Otro avance decisivo, en la misma dirección, ocurrirá en la década de 1960, con el aparecimiento de los sistemas de indexación, los cuales constituyen un supertexto, situado a un metanivel con respecto a las observaciones de primero y segundo orden de la ciencia (Leydesdorf \& Wouters, 1999). Tales sistemas fueron diseñados inicialmente por Eugene Garfield, quien es el fundador del Institute for Scientific Information (ISI), base del estándar hoy dominante para la evaluación del conocimiento científico que se publica (De Bellis, 2009).

Las citas llevan a cabo la labor selectiva y de enlazamiento propia del proceso de acumulación del conocimiento científico. La variedad no seleccionada desaparece y aquélla que muchos seleccionan positivamente se agrega al conocimiento aceptado. Los sistemas de indexación, que revisan y registran lo que es citado y lo difunden, ayudan a procesar las ingentes cantidades de textos científicos que aparecen periódicamente en el mundo y facilitan enfrentar su enorme proliferación. Las citas cumplen, así, esa función cognitiva fundamental para la reflexividad, selectividad y 
acumulación científica; pero, al mismo tiempo, las citas son indicadores de conexiones sociales dentro del campo, son indicadores de alianzas y de estrategias, existentes o buscadas. Las referencias bibliográficas dan luces sobre la organización social de las comunidades científicas (Leydesdorf \& Amsterdamska, 2000). En las citas aparecen las conexiones entre la dinámica del campo y la del sistema de comunicaciones. Se superponen en ellas las dimensiones social y cognitiva de la ciencia.

La cita a un texto dentro de una publicación científica es un mecanismo con más complejidad de la que aparenta. Hay cuatro aspectos de su uso y repercusiones performativas que es importante destacar. (1) Merton (1973) enfatizó su función de reconocimiento a los aportes de otros científicos $y$, de tal modo, como unidad básica del sistema de recompensas. Las citas otorgan crédito, el crédito simbólico que Bourdieu $(1988,2001)$ conceptualiza en términos de capital -capital científico-. (2) Esa función va directamente asociada al uso de las citas para constituir, dentro del texto publicado, el sustento para la investigación que en él se presenta: sustento teórico, en cuanto a conceptos y argumentaciones; sustento empírico, en cuanto a evidencias provenientes de otros estudios empíricos que sirven de apoyo; sustento metodológico, en cuanto a instrumentos o procedimientos elaborados o validados por otros autores. (3) Otra función destacada es persuadir al lector (Gilbert, 1977). Parte de la búsqueda de persuasión tendrá, naturalmente, que ver con las razones sustantivas indicadas en el punto previo, pero además, y a veces sin mayor conexión con ellas, se apela a las citas por razones del prestigio o legitimidad del autor o texto citado por el valor de reconocimiento histórico que un texto tenga en la comunidad científica -como puede ser el caso de Vigilar y Castigar, de Foucault, entre los sociólogos; o de Designing Social Inquiry, de King, Keohane y Verba, entre los cientistas políticos-. Ciertos textos venerados, de tal modo, aportan su aura a una nueva publicación, crean apariencia de que ésta cuenta con respaldo -teórico, empírico o metodológico- aún cuando se trate de mero uso retórico de la referencia bibliográfica. (4) Otro desdoblamiento de los anteriores usos y repercusiones performativas, que puede dirigir la construcción de las citas y referencias, es la de enrolar aliados (Callon \& Law, 1998), función que opera tanto textualmente como extratextualmente: las alianzas resultantes podrán manifestarse tanto al interior de nuevas publicaciones en que autores citados favorablemente hacen lo propio con el autor que los cita -le retribuyen con citas que apoyan sus argumentos o lo respaldan en contra de opositores comunes-. Estos enrolamientos a través de los textos pueden darse sin que en el intertanto haya ocurrido ningún contacto personal, como pueden 
estar también vinculados con colaboraciones o comunicaciones interpersonales en conferencias, congresos o actividades similares. La alianza así establecida puede ser de apoyo paradigmático, teórico, metodológico o evidencial, y también puede estar dirigida hacia la interface entre la ciencia social y los ámbitos de acción social y política no científicos.

Estos usos retóricos y de enrolamiento de las citas hacen que ellas, en muchas ocasiones, no sean realmente necesarias con respecto al contenido sustantivo de la investigación. Las citas con tal carácter prescindible o superficial respecto a la sustancia cognitiva del trabajo investigativo no son una proporción menor. Según algunos estudios, alcanzan entre el 30\% y el 40\% del total (Gilbert, 1977; De Bellis, 2009). De ellas, algunas ni siquiera provienen de una efectiva lectura del texto citado; para que el texto cumpla su función ritual de legitimación o de protección, basta con conocer el valor que tiene en la comunidad de referencia, en la audiencia de destino.

Junto con abrir posibilidades de alianzas futuras, las citas reflejan los vínculos y resultados preexistentes, dirigidos hacia una particular fracción de la comunidad científica (Gilbert, 1977); pueden darse paralela y complementariamente con vínculos sociales efectivos -pertenencia a una misma institución, inclusión en los mismos grupos de trabajo, vínculos informales, etc.-; o pueden operar exclusivamente en los textos, en la red de comunicaciones escrita y pública. Por otra parte, tales apoyos, enrolamientos y alianzas normalmente no son declarados en cuanto tales. Tienen un carácter tácito, pero son reconocibles e interpretables por los involucrados.

En todo caso, hay que enfatizar que los usos retórico y de enrolamiento para los que pueden ser empleadas las citas acompañan el uso sustantivo de ellas. Todos esos usos pueden darse simultánea y complementariamente; es así que, habitualmente, se busca enrolar a un autor cuyas investigaciones son claramente relevantes para la investigación que lo cita y se apela a autores teóricos prestigiosos, cuyas ideas efectivamente ayudan a la estructuración de la investigación en referencia a la cual son mencionados.

Todo lo anterior hace que cada científico tenga una "citation identity" (De Bellis, 2009: 247), una forma distintiva de citar, que responde a la interacción entre características del autor, de su formación y de características del campo disciplinar en que opera. Para resultados similares, de investigaciones similares, dos diferentes investigadores elaborarán conjuntos de citas que siguen pautas diferentes, que responden en buena medida a sus "identidades citacionales". 


\section{Publicaciones científicas y redes socioepistémicas}

Las relaciones entre citas y otros contenidos de los textos dan forma a una variedad de redes entre las publicaciones científicas que permiten explorar las estructuras subyacentes cognitivas y sociales. Para ello, se han constituido, desde los sesenta, diversos procedimientos de análisis, que han alcanzado mayor difusión y uso desde los años ochenta, gracias al desarrollo de software para el análisis de redes y a la creciente disponibilidad de bases de datos digitales.

Tales análisis de redes intertextuales consideran diferentes tipos de nodos: palabras, los textos completos, las revistas en que son publicados y los autores. Los tipos de análisis más destacados, respecto a las redes que se configuran entre dichos nodos, son el co-word analysis, co-citation analysis, análisis de referencias, mapas de influencia, y análisis de coautoría (Newmann, 2010; De Bellis, 2009; Gmür, 2003; Leydesdorff, 2001; Mutschke \& Quan, 2001; Hicks, 1987; Callon et al. 1983). Haremos una breve presentación de los tipos de redes y análisis, para especificar después los procedimientos que serán usados en este trabajo.

(1) Redes de palabras. En ellas, tal como lo considera el co-word analysis (Callon et al., 1983), los nodos son palabras y se considera que dos palabras están conectadas en la medida en que aparecen simultáneamente o "co-ocurren" en los mismos documentos. Una alta frecuencia de co-ocurrencias de un mismo par de palabras revela similitud de contenido entre ellas, vale decir, proximidad cognitiva. Las palabras analizadas pueden ser las keywords, o las palabras del título, o del abstract o las del texto completo o palabras clave identificadas por indexadores profesionales.

(2) Redes de textos. Los nodos son las diferentes publicaciones científicas de interés. En el co-citation analysis se considera que hay un vínculo entre dos publicaciones cuando ellas aparecen conjuntamente en las referencias bibliográficas de un mismo texto (Gmür, 2003).

(3) Redes de revistas. Los nodos son las revistas académicas y, en la lógica del cocitation analysis, se considera, tal como en el caso previo de los textos, que dos revistas están relacionadas cuando son mencionadas conjuntamente en las bibliografías de los mismos textos (Nelson, 2006).

(4) Redes de autores. Tal como en las redes anteriores, usando el co-citation analysis el vínculo entre autores puede establecerse sobre la base de su aparición conjunta en las referencias bibliográficas de los mismos documentos. Pero también puede considerarse la existencia de vínculo entre dos autores al ser uno citado por el 
otro (Hargens, 2000). Las redes de co-citación son simétricas, es decir sin direccionalidad. La red de referencias entre citantes y citados, en cambio, es asimétrica, posee dirección.

A través del trazado de las redes de palabras, textos o autores, es posible discernir clusters de conocimientos, establecer relaciones de proximidad entre unos y otros nodos, identificar tópicos, textos o autores centrales y vínculos entre ellos, así como elaborar mapas de influencias y explorar la evolución de temas científicos (Comtral \& Gordon, 1997; Hicks, 1987).

(5) Redes de coautoría. En este caso los nodos son autores y la conexión entre ellos se establece al ser coautores de un mismo texto (Rivellini et al. 2006; Mutschke \& Quan, 2001). En estas redes hay una clara explicitación de un vínculo de colaboración que ocurre en el plano extratextual. La red de coautoría corresponde a una efectiva red de colaboración social; el vínculo social no es inferido a través del texto, sino que declarado en él. ${ }^{3}$

Así como en las redes de coautoría hay un componente social que es destacado y en las redes de palabras un componente cognitivo predominante, en las otras hay diversas combinaciones entre ambos componentes. Los usos que antes anotábamos de las citas muestran precisamente esa doble dimensionalidad, que hace que las citas constituyan un mecanismo de acoplamiento entre el sistema de comunicaciones y el campo de relaciones y estrategias sociales. Son, así, redes socio-epistémicas o sociocognitivas.

Todas las mencionadas son redes "modo-1", es decir redes en que los nodos son del mismo tipo. Pero también se pueden trazar redes "modo-2", en las que se indaga en las relaciones entre dos conjuntos de nodos diferentes, por ejemplo, entre textos y autores, textos y revistas, o autores y revistas (De Nooy et al., 2005; Wasserman \& Faust, 1994).

Una perspectiva adicional es la que atiende a la evolución temporal de las redes. Suma al estudio de la topología de las redes la consideración de su macrodinámica, empleando datos longitudinales. Ello involucra analizar el surgimiento, transformación o disolución de redes y los procesos, a través del tiempo, de difusión, influencia, superposición y mímesis (Powell et al., 2005).

\footnotetext{
3 La coautoría refleja trabajo investigativo en común entre los coautores, aunque éste no incluya en particular el trabajo de escritura del texto en que aparecen como tales, el cual muchas veces es redactado sólo por algunos de los participantes.
} 


\section{Las comunicaciones en el campo de las ciencias sociales en Chile: Interrogantes de la investigación}

Algunas de tales redes y procedimientos son los que empleamos para estudiar el campo de las ciencias sociales en Chile, del cual consideramos tres disciplinas centrales: sociología, ciencia política y antropología social. Ya una primera pregunta a hacer es sobre la existencia misma de tal campo. ¿Existen efectivamente las interrelaciones comunicativas que permitan afirmar su existencia? ¿Se cuenta con una suficiente densidad de vínculos que atestigüe su presencia u operatividad? Entrevistas realizadas con investigadores del campo dan cuenta de fragmentación y debilidad en la constitución de la comunidad académica de las ciencias sociales, ¿ratifica el análisis de redes tales percepciones?

En cuanto a la estructura cognitivo-social del campo, que buscamos discernir, hay diversos factores que pueden incidir sobre la estructura cognitiva del campo. Indagaremos la incidencia de cuatro factores: las separaciones disciplinares, los marcos paradigmáticos en que encauzan su trabajo los investigadores, la pertenencia a diferentes instituciones de producción de conocimientos y el género de los investigadores.

En cuanto a las separaciones disciplinares, diversos planteamientos, como los de Wallerstein et al. (1996) hablan de corrientes de convergencia entre las disciplinas de las ciencias sociales y de la tendencia al desaparecimiento de sus fronteras. En un análisis de los conceptos empleados por las investigaciones de cada disciplina hemos constatado, en otro estudio, la mantención de claros perfilamientos disciplinarios, particularmente para el caso de la ciencia política (Ramos Canales \& Palestini, 2008). Tan sólo la antropología aparecía con un dominio conceptual más difuso. Sobre esa base, podemos hipotetizar que la estructuración cognitiva del campo y respectivas redes debería reflejar esa existencia de perfilamientos y fronteras entre las tres disciplinas.

Un segundo factor cuyo efecto sobre la forma de las interrelaciones cognitivas vamos a chequear son los paradigmas científicos en que se encuadra la labor de los investigadores. ${ }^{4}$ Los planteamientos originales de Kuhn (1971) enfatizaban la inconmensurabilidad de los paradigmas y su operación ineludiblemente separada. En el caso de las ciencias sociales, a diferencia de las ciencias estudiadas por Kuhn, conviven

\footnotetext{
${ }^{4}$ Usamos el concepto de paradigma científico de Kuhn, que remite al sustrato de supuestos ontológicos y epistemológicos sobre los que se sustentan las prácticas investigativas, situadas socioculturalmente.
} 
diferentes paradigmas $y$, además, esta convivencia ha sido ya prolongada en el tiempo, y se ha hecho más marcada y cercana desde los años 1960 en adelante. Esta situación hace que aumente la frecuencia de los casos de socialización de investigadores en más de uno de los paradigmas prevalecientes y que se lleven a cabo diversos procesos de traducción entre ellos. En investigaciones previas hemos encontrado que, de hecho, existe un grupo de investigadores a los cuales en términos paradigmáticos se podría calificar de híbridos, que emplean, de manera provechosa, combinaciones de elementos de uno y otro, o que pasan de una investigación encuadrable en un paradigma a una siguiente investigación encuadrable en otro (Ramos, 2005; Ramos \& Canales, 2009; Palestini, Ramos \& Canales, 2010) ${ }^{5}$. Aunque se produzca cierta disonancia epistemológica en tales investigaciones o investigadores, esas formas de investigación parecen constituir una perspectiva importante de trabajo científico social, que podría pensarse que provee gérmenes que a futuro -un futuro de plazo todavía indeterminado- podrán facilitar el enraizamiento de algún nuevo desarrollo paradigmático integrativo. Dado lo anterior, la pregunta que nos planteamos al respecto, es si los paradigmas, en el caso chileno, son efectivamente un factor diferenciador y estructurador de las comunicaciones científicas. Es decir, la pregunta es cuán diferenciadas se encontrarán las comunicaciones cuando se considera el encuadre paradigmático de los investigadores.

Los paradigmas y las configuraciones disciplinarias tienen relación directa con el proceso cognitivo, son parte suya. Otros factores que inciden sobre la estructuración de las comunicaciones científicas son, en cambio, externos a él. El ámbito institucional de producción de conocimientos es uno de estos factores externos. Las características de la red de comunicaciones puede estar condicionada por características de los centros de investigación. En todo campo científico hay algunas instituciones tradicionales que ocupan posiciones dominantes en el tejido de las comunicaciones y otras que están en posiciones subordinadas (Bourdieu, 1988). En el caso de Chile, durante la dictadura los centros académicos independientes se convirtieron en las principales fuentes de producción de conocimientos y desarrollaron múltiples conexiones entre sí y con fuentes internacionales de financiamiento (Garretón, 2005). En el período post-dictadura, a partir de 1990, las universidades han vuelto a tomar posiciones de importancia en la producción científico social. Sin embargo, como derivado de las transformaciones en la institucionalidad universitaria ocurridas bajo el

\footnotetext{
${ }^{5}$ En tales textos, además, hacemos una descripción y fundamentación de los encuadres paradigmáticos y explicamos de qué forma fueron realizadas las clasificaciones de los textos y sus autores.
} 
régimen autoritario, junto a las antiguas grandes universidades tradicionales, han aparecido las privadas, algunas de las cuales le han prestado particular atención a la investigación, especialmente a la de relativo bajo costo, como es el caso de la investigación en ciencias sociales. Por tanto, lo que buscamos averiguar al respecto es cómo se expresa la variedad de instituciones en la estructura de comunicaciones. ¿Siguen ocupando posiciones centrales los centros académicos independientes? ¿Han sido reemplazados por las universidades tradicionales que han retornado a sus posiciones de privilegio? ¿Qué lugar ocupan las universidades privadas? También nos preguntamos por la posición relativa entre instituciones metropolitanas versus regionales. En cuanto a esto último, dada la tradición centralista, cabe plantearse la hipótesis de que las fuentes de producción regional ocuparán posiciones periféricas en la red de comunicaciones. Sería inesperado que no ocurriera así.

Un segundo factor "externo" que consideraremos es el género. La ciencia durante mucho tiempo fue terreno predominantemente masculino. Aunque esto ha ido cambiando, tal cambio ha ocurrido lentamente. ${ }^{6}$ Frente a esto, cabe preguntarse cómo se encuentran posicionadas las mujeres en la red de comunicaciones? ¿Qué características estructurales tienen sus relaciones comunicativas?

Además de redes cuyo entrelazamiento ocurre directamente en los textos, estudiaremos una red social que toma lugar directamente en la acción investigativa: la red de colaboración manifestada en coautorías y nos preguntamos por las características que asumen en el país las estructuras de colaboración científica. A través del mundo, el trabajo científico en colaboración ha ido aumentando significativamente en las últimas décadas, especialmente en ciencias como la química o la física, donde el porcentaje de publicación en coautoría supera el 70\% (Haan, 1997). En ciencias sociales parece estar entre el 30 y 40\% (Gingras, 2002), aunque otras estimaciones ponen cotas más altas (Larivière, Gingras \& Archambaul, 2006). Particularmente destacado ha sido el aumento de la colaboración internacional. El año 2000 , se estima que el $15.6 \%$ de las publicaciones ISI habría sido en coautoría internacional (Wagner \& Leydesdorff, 2004). En ciencias sociales la cifra sería algo menor; para el período 1995-1997, Gingras (2002) la estima en un 8.6\% a nivel global y en $10.3 \%$ para USA. Respecto a un país periférico, Sudáfrica, Mouton (2010) calcula que, para el período 2000 a 2004, un $10 \%$ de las publicaciones de la sociología fue

\footnotetext{
${ }^{6}$ De los textos provenientes de la ciencia política que integraban nuestro corpus de estudio, el $72 \%$ tenía un hombre como autor o, cuando tenía más de un autor, había mayoría masculina (Ramos \& Canales, 2009).
} 
escrita en coautoría con extranjeros, lo que contrasta con el $2.8 \%$ del período 1990 1992, para el mismo país. Estas cifras sirven de referencia comparativa para preguntarse por la situación de la colaboración tanto local como internacional de las publicaciones realizadas por los investigadores del campo de las ciencias sociales en Chile.

En cuanto a tal colaboración internacional, en Chile ha sido reciente la preocupación por establecer dichas conexiones y tan sólo en la década del 2000 el Consejo Nacional de Ciencia y Tecnología (Conicyt) ha puesto énfasis en promover iniciativas de colaboración internacional el cual se ha visto manifestado en apoyos financieros significativos y en gestiones para facilitar contactos internacionales. Sus efectos cabe esperar que se comiencen a notar principalmente hacia el fin de tal década y poco en los años estudiados. Tampoco ha sido usual, hasta hace poco, dentro de los departamentos de ciencias sociales en las universidades, incentivar tal tipo de colaboración. Por tanto, cabe esperar una baja cifra de colaboración internacional.

Las coautorías conectan a los investigadores dentro del campo. Entrevistas que hemos realizado a investigadores nacionales revelan gran desconocimiento sobre la producción científico social en áreas diferentes a la propia. De ser esto generalizado, cabría esperar que las colaboraciones no conectaran sectores importantes del conjunto de nodos del campo y que las conexiones fueran fragmentarias. En redes altamente conectadas (como la Web) un componente de la red incluye conexiones a más del $50 \%$ de los nodos (Newman, Barabási \& Watts, 2006). Hipotéticamente planteamos que ese no sería el caso de esta red de colaboración; ésta no sería una red altamente conectada. Por otra parte, la colaboración podría ser una estrategia de las mujeres para compensar sus dificultades de inserción en el campo científico. Esto hace relevante averiguar cómo se insertan las mujeres en relaciones de colaboración.

\section{Metodología}

En esta investigación empírica, uso las redes intertextuales y las redes de coautoría para inferir características de las estructuras de relación en el campo de la ciencia social. Nuestra base empírica son las publicaciones científicas y nuestra herramienta analítica el Análisis de Redes. El análisis de las redes de referencias bibliográficas, en el sistema de las comunicaciones científicas, provee pistas sobre la estructura de las relaciones en el campo. Las redes de coautoría, por su parte, revelan de modo más directo las pautas de entrelazamiento de los investigadores. 
El foco del estudio está en la ciencia social que se produce en el período presente en el país y en los investigadores que generan nuevos conocimientos. He considerado tres disciplinas fundamentales de las ciencias sociales: sociología, ciencia política y antropología social.

Las investigaciones que abordan redes de referencias, usualmente atienden sólo a las publicaciones contenidas en revistas, por razones de accesibilidad. Esto es válido en ciencias como la química o la biología, donde la mayor parte de los resultados del trabajo investigativo aparece efectivamente en artículos de revistas. Pero en las ciencias sociales la pauta de publicación es diferente. En particular, la sociología ha sido una cultura del libro, como lo han mostrado Clemens et al. (1995) con respecto a USA. Por tanto, no hemos aplicado tal restricción, eventualmente distorsionadora, y hemos cubierto los diferentes formatos habituales de publicación: libros, capítulos de libros, artículos de revistas y documentos de trabajo con difusión pública.

El requisito principal de selección de textos ha sido que ellos correspondan al núcleo dinámico del proceso científico, que contengan conocimiento nuevo generado a través de trabajo sistemático de investigación. Esto incluye la generación de conocimiento abstracto o teórico. Se excluyen los book reviews, los artículos de opinión y textos que no reúnan los requisitos mínimos para ser seleccionados en una revista científico social de calidad reconocida. Para seleccionar un texto, su autor -o al menos uno de los autores, en los casos de autores múltiples- debía tener formación de pre o postgrado en una de las tres disciplinas consideradas. Se consideró como integrantes del campo no sólo a investigadores de nacionalidad chilena, sino también a aquéllos que tuvieron residencia estable en Chile durante el período estudiado y participaron activamente en el campo.

La investigación pretendió cubrir el universo de textos que reunieran los requisitos anotados, durante el período 2000 a 2006. Se prestó especial atención a recoger material producido en regiones y se recorrieron centros regionales pesquisando textos. También revisamos diversas series de documentos de trabajo y publicaciones de instituciones, públicas, privadas e internacionales, y solicitamos sus publicaciones a académicos que podían tenerlas y que no habíamos encontrado. En cuanto a publicaciones realizadas en revistas extranjeras, revisamos revistas ISI y Scielo de ciencias sociales, buscamos a autores nacionales que escribieran en ellas y revisamos si cumplían los requisitos exigidos. Todo ello constituyó un significativo esfuerzo por lograr el máximo de cobertura, de tal modo que los textos que finalmente no hayan sido identificados y encontrados, aún debiendo haber estado en el corpus, deberían 
representar una fracción reducida y no hay razones para pensar que haya algún sesgo en su exclusión; su distribución debería ser aleatoria. Finalmente, quedó constituido un corpus de 479 textos. En la tabla siguiente está la distribución de tales publicaciones, clasificadas según disciplina de los autores y tipo de formato.

\begin{tabular}{|c|c|c|c|c|c|c|}
\hline & & \multicolumn{5}{|c|}{ DISCIPLINAS } \\
\cline { 2 - 7 } FORMATOS & TOTAL & Sociología & $\begin{array}{c}\text { Ciencia } \\
\text { Política }\end{array}$ & $\begin{array}{c}\text { Antropo- } \\
\text { logía }\end{array}$ & $\begin{array}{c}\text { Sociología } \\
\text { \& Cs. Pol. }\end{array}$ & $\begin{array}{c}\text { Sociología } \\
\text { \& Antrop. }\end{array}$ \\
\hline $\begin{array}{c}\text { Artículo de } \\
\text { revista }\end{array}$ & 42.4 & 29.3 & 53.3 & 53.3 & 43.3 & 54.8 \\
\hline $\begin{array}{c}\text { Libro o capítulo } \\
\text { de libro }\end{array}$ & 35.9 & 50.9 & 21.5 & 29.3 & 33.3 & 16.2 \\
\hline $\begin{array}{c}\text { Documento de } \\
\text { trabajo o } \\
\text { formato similar }\end{array}$ & 21.7 & 19.7 & 25.1 & 17.4 & 23.3 & 29.1 \\
\hline & $\begin{array}{c}100.0 \\
(479)\end{array}$ & $\begin{array}{c}100.0 \\
(208)\end{array}$ & $\begin{array}{c}100.0 \\
(135)\end{array}$ & $\begin{array}{c}100.0 \\
(75)\end{array}$ & $\begin{array}{c}100.0 \\
(30)\end{array}$ & $\begin{array}{c}100.0 \\
(31)\end{array}$ \\
\hline
\end{tabular}

Tabla 1: Distribución de las publicaciones seleccionadas según formato y disciplina (en \%)

Estas cifras reflejan la importancia que tiene en Chile el libro como medio de difusión de conocimientos dentro de las ciencias sociales y especialmente en la sociología, en concordancia con lo encontrado con respecto a USA (Clemens et al., 1995). Esto ratifica el criterio previamente establecido de no excluir este formato de publicación.

Los análisis de referencias bibliográficas y co-citaciones por lo general sólo registran al primer autor de una publicación, lo cual implica una considerable pérdida de información (Porter, 1977; Gmür, 2003) y es una limitación de importancia si se quiere estudiar las redes de comunicación entre investigadores, como es nuestro caso. En consecuencia, no nos hemos limitado al primer autor sino que hemos considerado también a los co-autores. ${ }^{7}$

Cada uno de los 479 textos del corpus fue sometido a un análisis que combinó tanto procedimientos interpretativos como cuantitativos y que permitió caracterizarlo en cuanto a su marco paradigmático. Luego de una lectura y revisión exhaustiva de cada texto, lo clasificamos de acuerdo a siete variables -postura epistemológica expresada en el texto, sentido de verdad, presencia explícita de valores, índice de ingerencia de valores en la investigación científica, atribución de carácter objetivo o significativo a la

\footnotetext{
${ }^{7}$ Hasta un máximo de 5.
} 
realidad, índice de positivismo metodológico e índice de desarrollo metodológico interpretativo- que luego empleamos en un procedimiento de cluster analysis para tipificar el encuadramiento paradigmático de la investigación reportada en el texto. Sobre esa base, distinguimos entre encuadres positivistas (en una variante más compleja que Ilamamos "fuerte" y otra "débil"), interpretativos e híbridos (que combinan rasgos de ambos); la orientación crítica, por su parte, estaba diluída entre estos dos últimos, y no la diferenciamos. ${ }^{8}$ Apelando a informaciones contenidas en las mismas publicaciones y usando otras fuentes, cuando fue necesario, especificamos diversos atributos de los actores: institución (principal) de pertenencia en el período estudiado, grado académico más alto alcanzado y género. Por otra parte, para cada texto se hizo el registro de todas sus referencias bibliográficas, incluyendo para cada cita todos los datos reportados por el autor. Después de un trabajo largo y muy demoroso -más allá de todas nuestras estimaciones- obtuvimos un total de $\mathbf{2 1 . 7 8 7}$ referencias bibliográficas. $^{9}$

Para indagar sobre la estructura de las relaciones sociocognitivas en el campo de las ciencias sociales analizamos redes de referencias y redes de coautorías.

(1) Sobre la base de las citas y referencias presentes en los textos del corpus construimos y analizamos la red direccional de investigadores, tanto la red global del campo como las redes de cada una de las tres disciplinas. La red del campo, considerando a todos los autores nacionales involucrados en los textos consiste de 579 nodos. Las redes disciplinarias, en las cuales incluimos solamente aquellos autores del corpus pertenecientes a las tres disciplinas estudiadas, quedó compuesta por 205 investigadores en sociología, 91 en ciencia política y 65 en antropología.

(2) Considerando las coautorías presentes en el corpus textual, elaboramos otra red de investigadores. En este caso, los lazos de la red se constituyen por la colaboración entre dos o más investigadores, expresada en ser coautores de un texto. Excluyendo a los investigadores que sólo son autores individuales de textos, la red quedó constituida por 312 nodos.

Este conjunto de redes lo hemos estudiado a través del Análisis de Redes Sociales (Wasserman \& Faust, 1994; Scott, 2000; Degenne \& Forsé, 2004), usando diferentes

\footnotetext{
${ }^{8}$ Para mayor explicación del procedimiento, ver Ramos et al. (2008) y Ramos (2005).

${ }^{9}$ La cantidad total de referencias registradas es en realidad mayor, pero para objeto del presente análisis hemos descartado las referencias a diarios y a los sitios web en que no se alude a un texto con un autor personal $o$ institucional- identificable. De cada referencia identificamos sus diferentes características como atributos para el posterior análisis.
} 
programas computacionales: ORA, UCINET y Pajek. ${ }^{10} \mathrm{~A}$ través de ellos hemos calculado diferentes medidas sobre la topología de la red, en cuanto a su estructura y a características posicionales de los nodos. Como parte del análisis, además, es muy importante la visualización de la red. Pajek incluye algoritmos que permiten una representación robusta de los datos de redes en un espacio euclideano bidimensional. En particular, para el análisis visual hemos empleado dos procedimientos que permiten optimizar la imagen de la red: el algoritmo de Fruchterman Reingold que separa claramente las partes no conectadas de la red y el algoritmo de Kamada-Kuwai que reposiciona los nodos conectados haciendo que las distancias euclideanas entre los nodos sean regularmente proporcionales a las distancias registradas en las matrices de base, hasta llegar a una situación estable (De Nooy et al., 2005).

Frente a la complejidad de redes de gran tamaño, la visualización que permiten los actuales programas es una gran ayuda para discernir las pautas de entrelazamiento. Por tal razón apelamos a ellas y agregamos al informe algunos gráficos particularmente ilustrativos, aunque en el papel se pierda la nitidez que tales gráficos tienen en la pantalla del computador y desaparezcan las posibilidades de activar la imagen ("abrir" nodos para ver sus características, mover grupos de nodos, hacer mediciones estructurales y aplicarlas a la imagen en pantalla, etc.).

A continuación, exponemos los resultados obtenidos.

\section{Redes en el campo de las ciencias sociales de Chile}

\section{Distribución de las comunicaciones}

Como primera aproximación a las pautas de comunicación, mostraremos la distribución de las referencias bibliográficas de acuerdo a las características de los autores citados, considerando tres aspectos de éstos: disciplina, carácter institucional o no y región en donde hace su trabajo. En la Tabla 2 aparece tal distribución de las referencias.

\footnotetext{
${ }^{10}$ UCINET y Pajek son herramientas ampliamente difundidas en el análisis de redes. ORA es un software desarrollado en la Carnegie Mellon University.
} 


\begin{tabular}{|c|c|c|c|c|c|}
\hline \multirow{2}{*}{\multicolumn{2}{|c|}{$\begin{array}{l}\text { CARACTERISTICAS DEL } \\
\text { AUTOR CITADO } \\
\text { (Disciplina / país de } \\
\text { procedencia) }\end{array}$}} & \multicolumn{3}{|c|}{ DISCIPLINA DEL AUTOR CITANTE } & \multirow{3}{*}{$\begin{array}{c}\text { TOTAL } \\
7.7\end{array}$} \\
\hline & & \multirow{2}{*}{$\frac{\text { Sociología }}{12.1}$} & \multirow{2}{*}{$\begin{array}{c}\begin{array}{c}\text { Ciencia } \\
\text { Política }\end{array} \\
2.0\end{array}$} & \multirow{2}{*}{$\begin{array}{c}\begin{array}{c}\text { Antropo- } \\
\text { logía }\end{array} \\
4.3\end{array}$} & \\
\hline \multirow{7}{*}{ CHILE } & Sociólogos & & & & \\
\hline & $\begin{array}{c}\text { Cientistas Polí- } \\
\text { ticos }\end{array}$ & 0.8 & 7.3 & 0.6 & 3.0 \\
\hline & Antropólogos & 0.8 & 0.1 & 9.7 & 1.7 \\
\hline & $\begin{array}{l}\text { Organismos } \\
\text { estatales }\end{array}$ & 5.3 & 4.6 & 1.7 & 4.6 \\
\hline & $\begin{array}{c}\text { Organismos } \\
\text { internacionales }\end{array}$ & 1.7 & 0.6 & 0.3 & 1.1 \\
\hline & $\begin{array}{c}\text { Centros } \\
\text { Independientes }\end{array}$ & 1.6 & 0.7 & 0.6 & 1.1 \\
\hline & $\begin{array}{c}\text { Otros autores no } \\
\text { corpus ni vinc. al } \\
\text { corpus }\end{array}$ & 22.3 & 23.9 & 26.0 & 23.3 \\
\hline CHILE & $\begin{array}{c}\text { Total autores } \\
\text { chilenos }\end{array}$ & 44.6 & 39.2 & 43.2 & 42.6 \\
\hline \multicolumn{2}{|c|}{ OTRO PAIS AMÉRICA LATINA } & 10.7 & 10.2 & 12.7 & 10.8 \\
\hline \multicolumn{2}{|c|}{ USA / CANADA } & 14.1 & 27.8 & 11.9 & 18.5 \\
\hline \multicolumn{2}{|c|}{$\begin{array}{c}\text { EUROPA (UK, Francia, Alemania, } \\
\text { España, otro país europeo) }\end{array}$} & 25.1 & 21.0 & 26.5 & 24.0 \\
\hline \multicolumn{2}{|c|}{$\begin{array}{c}\text { OTRO PAIS, VARIOS PAISES } \\
\text { O PAIS NO IDENTIFICADO }\end{array}$} & 5.4 & 1.8 & 5.7 & 4.2 \\
\hline \multicolumn{2}{|r|}{ TOTAL } & $\begin{array}{c}100.0 \\
(11.582)\end{array}$ & $\begin{array}{l}100.0 \\
(7.369)\end{array}$ & $\begin{array}{l}100.0 \\
(2.836)\end{array}$ & $\begin{array}{c}100.0 \\
(21.787)\end{array}$ \\
\hline
\end{tabular}

Tabla 2: Distribución por disciplina, tipo de fuente y país de las referencias biblio-gráficas, según disciplina del autor que cita (distribución de los autores citados en \%)

Al observar las comunicaciones internas en el campo nacional se destaca que es particularmente baja la proporción de referencias que los investigadores del corpus hacen a otros investigadores científicos - de la sociología, ciencia política y antropología- que están actualmente investigando y publicando. En la Tabla 2, las tres primeras filas corresponden a tales investigadores activos en el período estudiado, en contraposición a otros investigadores (que, por ejemplo, publicaron en períodos previos), los cuales están clasificados como "autores no corpus". Sólo un $12.4 \%$ del total de las citas va a tales investigadores activos en el período 2000-2006, siendo los cientistas políticos los que menos citan a sus colegas investigadores (en el caso de los sociólogos tales citas a investigadores activos son un 13.7\%; en los cientistas políticos un $9.4 \%$ y en los antropólogos un $14.6 \%$ ). Esto puede leerse como un indicador del 
poco aprovechamiento del trabajo de los pares - por desconocimiento, problemas de difusión y otros factores-. El análisis de redes nos pemitirá profundizar en esta situación de desconexión, que la mera distribución de citas no permite.Los resultados obtenidos resaltaron la relevancia que tienen los autores institucionales, de textos, en que no se identifican autores individuales. Hay un importante uso de documentos, informes de datos de fuentes estatales, especialmente en el caso de la sociología y ciencia política, el cual es parte de una sinergia que ha aumentado en las últimas décadas entre el Estado y la ciencia social (ver Ramos, 2008).

Se observa una proporción relativamente parecida de citas que van hacia el interior del país o del ámbito latinoamericano en comparación a las que van a países del resto del mundo, principalmente países de capitalismo avanzado (53.4\% vs. $46.7 \%$ ). América Latina, sin embargo, no aparece como un foco destacado para las referencias, en ninguna de las tres disciplinas: recibe sólo un $10.8 \%$ del total.

\section{Características generales de la estructura de las redes de comunicación}

Para construir las redes de comunicación hemos considerado a los investigadores como nodos y a las refererencias bibliográficas que un autor hace a otro como vínculos direccionales del citante al citado. Para la constitución de cada una de estas redes se elaboraron matrices cuadradas, siendo analizados los vínculos de todos los nodos de cada red entre sí. El análisis se referirá tanto a la red total del campo como a las subredes disciplinares.

En el análisis hemos revisado diferentes niveles de configuración de las redes y subredes. (1) Un nivel de observación corresponde a las tres redes disciplinares, vistas por separado; cada una compuesta exclusivamente por autores de textos del corpus que sean integrantes de la disciplina: en sociología 205 integrantes, en ciencia política 91 y en antropología 65. (2) Otro nivel es el de cada subred disciplinar, compuesta de autores del corpus, vista como formando parte del conjunto de las tres redes, es decir como parte de una red con 361 nodos. (3) Un tercer nivel es el de la red total, compuesta no sólo de los integrantes de las tres disciplinas, sino también autores del corpus correspondientes a otras disciplinas como psicología, economía o historia, e incluyendo a autores nacionales de las obras citadas, que no sean autores del corpus; estos últimos sólo tienen conexiones de entrada, lo cual obliga a tratar la red como simétrica. En este caso, el total de nodos alcanza a 579.

En la Tabla 3 se incluyen varias medidas de las redes estudiadas que nos permiten caracterizar su estructura y que revisaremos a continuación. 


\begin{tabular}{|c|c|c|c|c|}
\hline \multirow[b]{2}{*}{ MEDIDAS DE RED (*) } & \multirow[b]{2}{*}{ RED TOTAL } & \multicolumn{3}{|c|}{ DISCIPLINA } \\
\hline & & SOCIOLOGIA & $\begin{array}{l}\text { CIENCIA } \\
\text { POLITICA }\end{array}$ & $\begin{array}{l}\text { ANTRO- } \\
\text { POLOGIA }\end{array}$ \\
\hline Cantidad de nodos & 579 & 205 & 91 & 65 \\
\hline Arcos & 5914 & 2247 & 644 & 307 \\
\hline Loops & 790 & 497 & 221 & 19 \\
\hline$\%$ de loops & 13.4 & 12.1 & 34.3 & 6.2 \\
\hline Densidad de la red & 0.006 & 0.012 & 0.012 & 0.010 \\
\hline $\begin{array}{l}\text { Densidad red disciplinar } \\
\text { respecto red integrada (361 } \\
\text { nodos) }\end{array}$ & - & 0.008 & 0.002 & 0.001 \\
\hline Ambito de control & - & 6.164 & 3.478 & 2.469 \\
\hline Reciprocidad & - & 0.08 & 0.05 & 0.01 \\
\hline Eficiencia global & 0.332 & 0.361 & 0.299 & 0.292 \\
\hline $\begin{array}{l}\text { Eficiencia global respecto a } \\
\text { red integrada ( } 361 \text { nodos) }\end{array}$ & - & 0.116 & 0.019 & 0.009 \\
\hline $\begin{array}{c}\text { Jerarquía } \\
\text { (Krackhardt) }\end{array}$ & 0.888 & 0.886 & 0.961 & 0.970 \\
\hline $\begin{array}{c}\text { Centralización de } \\
\text { intermediación }\end{array}$ & 0.076 & 0.118 & 0.088 & 0.112 \\
\hline $\begin{array}{c}\text { Coeficiente de } \\
\text { conglomeración (Watts- } \\
\text { Strogatz) }\end{array}$ & 0.216 & 0.232 & 0.193 & 0.146 \\
\hline Speed, average & 0.257 & 0.309 & 0.282 & 0.312 \\
\hline
\end{tabular}

(*) Nota: En algunos casos, cuando se indica, se considera a cada red disciplinar junto a las otras dos, por tanto contando con un total de 361 nodos.

Tabla 3: Características de las redes de referencias entre los científicos sociales (medidas referidas a la red global del campo y a las redes disciplinarias por separado)

La red de referencias muestra una proporción importante de loops o "lazos reflexivos" (Wasserman \& Faust, 1997: 95), es decir, de autores que se citan a sí mismos: un $13.4 \%$ del total de vínculos son loops. La proporción es particularmente alta en ciencia política, con $34.3 \%$ y en sociología, con $22.1 \%$, mientras en antropología es sólo 6.2\%. Según algunas estimaciones, el promedio general, a nivel internacional, de las autocitas estaría en torno al 10\% (Porter, 1977), según lo cual la situación en el campo chileno sería de un excesivo uso de la autoreferencia, lo cual probablemente indique que una proporción importante de ellas se esté usando con fines no exclusivamente sustantivos sino que además o principalmente como práctica de visibilización y legitimación del autor en el campo. 
El coeficiente de densidad permite constatar que la densidad de las comunicaciones científicas dentro del campo de las ciencias sociales es baja. Este coeficiente que mide la proporción de conexiones de una red en referencia al máximo posible de conexiones en esa red (Wasserman \& Faust, 1994), oscila entre 0 y 1 . En las redes estudiadas el valor es inferior a 0.03 , reflejando que menos del $3 \%$ de las conexiones posibles se concreta efectivamente. Cuando se consideran estas tres redes juntas, la densidad baja sustancialmente; esto era previsible, pero la baja es particularmente marcada en la antropología y ciencia política, reflejando su menor conexión con el campo en su conjunto, en comparación con la sociología.

Una medida que nos arroja un resultado complementario es la eficiencia global (Latora \& Marchiori, 2001). Este coeficiente, que también varía entre 0 y 1, refleja la cercanía de las entidades en la red, considerando las distancias geodésicas (caminos más cortos) entre todos los pares de nodos. Nuevamente, los valores son relativamente bajos. Tan sólo en la sociología llegan a más de 0.3.

El Ilamado ámbito de control agrega otra forma de cuantificar el bajo grado de conexión existente. Indica el promedio de conexiones de salida por nodo que tienen out degrees diferentes de cero, vale decir que tienen a su vez conexiones a otros nodos. Así, por ejemplo en la antropología, un investigador, en promedio, hace referencias tan sólo a 2.5 otros antropólogos nacionales, activos en el período reciente, que a su vez citan a otros investigadores nacionales de su propia disciplina (activos en el período reciente). Esta cifra sube a 3.5 en la ciencia política y a 6.2 en la sociología.

La reciprocidad de las citas, en la red global y en las redes disciplinares, también es baja. Esta medida corresponde al porcentaje de vínculos que son recíprocos, sin contar las autoreferencias (Carley, 2002). Los valores oscilan entre 0.08 para la sociología y 0.01 para la antropología. Estas cifras señalan que, para el caso de la sociología tan sólo un $8 \%$ de los investigadores hace referencia a otros investigadores que a su vez hacen referencia a ellos; en el caso de la ciencia política ello ocurre con el $5 \%$ y en la antropología con el $1 \%$ de los investigadores. Esta no reciprocidad en la gran mayoría de las citas reflejas pautas jerárquicas en la constitución del campo. En los términos de Bourdieu (1999) indica la presencia de unos pocos científicos en posiciones dominantes, poseedores de gran stock de capital simbólico, y un gran número en posiciones subordinadas. Estos resultados se ven complementados por los que proporciona el coeficiente de jerarquía (Krackhardt, 1994). Esta medida ratifica que 
las redes estudiadas muestran en alto grado una estructura jerárquica, especialmente la ciencia política. A su vez, la red presenta bajos valores de centralización de intermediación. Esta medida se basa en los puntajes de centralidad de intermediación, en la capacidad de conectar sectores diferentes de la red, que tiene cada investigador.

En clara concordancia con el conjunto de los resultados anteriores está el clustering coefficient (Watts-Strogatz, 2006). Este coeficiente mide el grado de conglomeración de una red promediando el coeficiente de conglomeración de cada nodo. El coeficiente de conglomeración de un nodo es la densidad de su ego network ("red egocéntrica"), que es el subgrafo derivado de sus vecinos inmediatos. A nivel de la red total, el coeficiente de conglomeración es el promedio de la proporción de links entre cada investigador y sus vecinos directos, dividido por el número de conexiones que podrían existir entre ellos. Este coeficiente, que tal como los otros varía entre 0 y 1 , es bajo en todas las redes disciplinarias y en el conjunto. Este coeficiente de conglomeración da una apreciación de las características locales de la red: cómo la información se difunde por medio de los grupos de investigadores. Un alto valor en el coeficiente de conglomeración refleja difusión local de información tanto como una distribución descentralizada.

El coeficiente Speed, promedio, es la inversa de la distancia geodésica entre todos los pares de nodos. El puntaje más alto es alcanzado por una clique y el más bajo por los nodos aislados. Es el promedio de la inversa de la centralidad de cercanía para los nodos de la red (Carley, 2002). Capta cuán rápidamente la comunicación fluirá a través de la red. En concordancia con el conjunto de medidas anteriores, los resultados son relativamente bajos.

La representación gráfica de las redes estudiadas, realizada por los antes mencionados programas de análisis, permite visualizar tales características de baja densidad, jerarquía, etc. Son muchos los gráficos ilustrativos que podríamos incluir, sin embargo nos vamos a concentrar en algunos que son sustantivamente más significativos y que agregan información a lo ya dicho por las medidas cuantitativas. Particularmente, analizaremos el eventual efecto separador o diferenciador que tienen los factores cuya influencia hemos hipotetizado: disciplinas, marcos paradigmáticos, pertenencia institucional y género, para luego analizar la red de conexiones teóricas en el campo.

\section{La red de comunicaciones global y las disciplinas}


La red total se presenta en el Gráfico 1. Una primera apreciación frente a él es que la extensión y cobertura del tejido de la red trasluce la existencia efectiva de un campo científico. El conocimiento producido por ese conjunto de nodos está suficientemente interconectado como para señalar el perfilamiento del campo de las ciencias sociales. Pero, junto a eso, la integración de éste no es fuerte; se observa dispersión y fragmentación. Existen diversos conjuntos de investigadores que están muy apartados e incomunicados entre sí y hay pocos investigadores que conecten las zonas apartadas del campo.

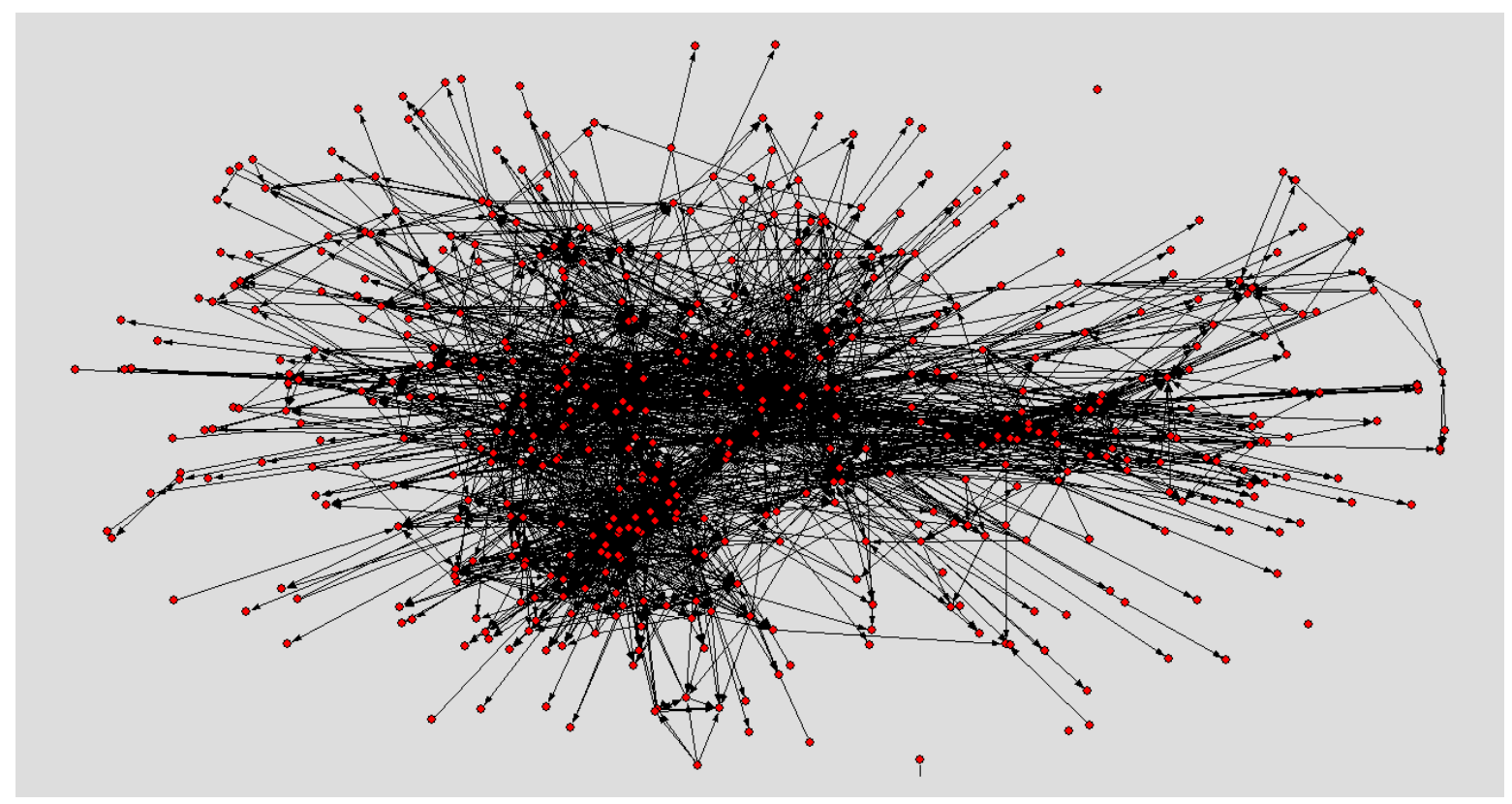

Gráfico 1: Red de comunicaciones del campo total.

En el Gráfico 2 aparece la red global, diferenciándose en ella los nodos según su pertenencia disciplinaria. En rojo están los nodos de sociología, en amarillo los de ciencia política, en azul los de antropología y en gris los de otras disciplinas.

La constitución disciplinar del campo permite dar cuenta de los principales focos de conglomeración y fragmentación de la red global. 


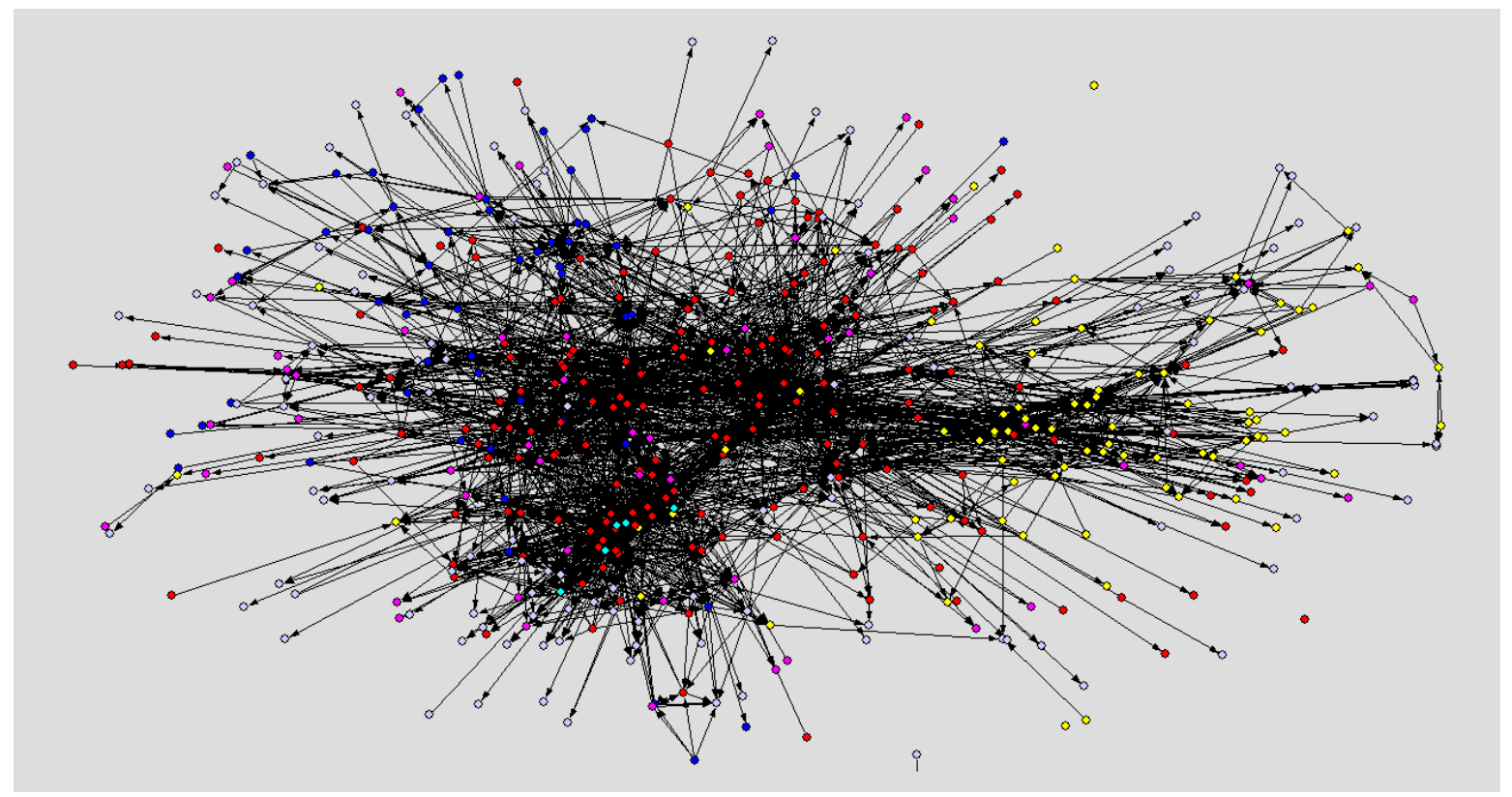

Gráfico 2: Red de comunicaciones del campo total, considerando disciplinas.

Puede observarse, en el gráfico anterior, que hay una clara división entre las redes de las tres disciplinas. Dos zonas aparecen con un nítido perfilamiento disciplinar. El centro del campo lo ocupa la sociología (nodos rojos), reflejando su mayor centralidad en la estructura de conexiones de la red global. A la derecha, con una clara preferencia por las conexiones intradisciplinarias, está la ciencia política (nodos amarillos). Algunos nodos amarillos en medio de otras disciplinas corresponden a investigadores con especiales funciones de enlazamiento entre disciplinas; operan como brokers o mediadores entre ellas. Es el caso de Norbert Lechner, conspicuo nodo amarillo en medio de rojos. Algunos nodos azules en el espacio rojo también son especiales en su rol de intermediación.

La antropología (nodos azules) está situada sobre todo en el sector superior izquierdo pero tiene una presencia menos compacta, con conexiones más dispersas. El gráfico muestra claramente su total desconexión de la ciencia política. La diferenciación conceptual y definido perfilamiento semántico de la sociología y ciencia política, que habíamos encontrado al analizar los conceptos empleados en los textos del corpus, junto al relativo desperfilamiento conceptual de la antropología (Ramos et al., 2008), encuentra su contrapartida en las configuraciones de red dentro del campo. Del mismo modo, lo que concluíamos en tal análisis de las estructuras conceptuales de las disciplinas, en cuanto al acercamiento conceptual entre la antropología y la sociología y 
al distanciamiento con la ciencia política, aquí ve su correlato en las redes de los investigadores (amarillo y azul situados en regiones opuestas), lo cual se repite por más iteraciones que hagamos en la graficación, optimizando las posiciones: siempre quedan en zonas opuestas y separadas.

Los nodos grises son investigadores de otras disciplinas y están entremezclados en la red total, sin coherencia entre sí, lo cual es concordante con el hecho de que ellos corresponden a un conjunto heterogéneo que incluye representantes de la historia, arquitectura, derecho, psicología, ingeniería, etc. Pese a todo, sin embargo, hay una relativa mayor concentración de estos nodos grises en la sociología, dentro de la nube roja, lo cual podría indicar una mayor receptividad a ellos en esta disciplina.

Es destacable la posición central de la sociología, conectando a la ciencia política y antropología. Sin la presencia de la red de sociólogos, estas dos disciplinas quedarían prácticamente desconectadas. Sin esa red mediadora el campo desaparece.

\section{Redes de acuerdo a paradigmas científico-sociales}

El efecto diferenciador de los paradigmas científicos sobre las comunicaciones lo revisaremos por disciplina, al interior de las cuales se perfila con mayor nitidez. La incidencia que tienen los encuadramientos paradigmáticos de los investigadores sobre la configuración de la red de sociología se puede visualizar en el Gráfico 3.

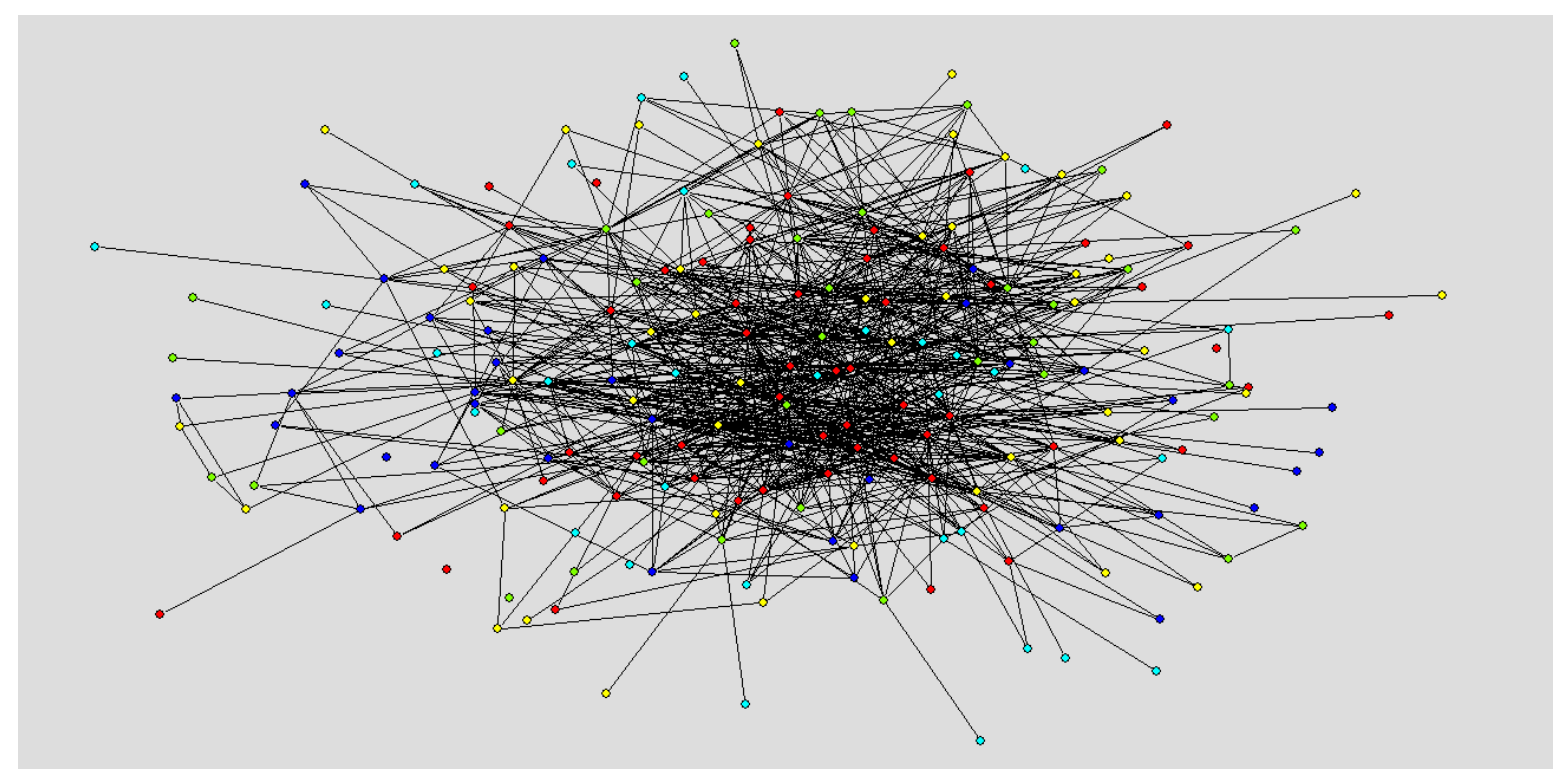

Gráfico 3: Red de comunicación de la sociología, considerando los paradigmas. 
En esta red, los paradigmas no constituyen una barrera infranqueable en términos de comunicaciones académicas. Entre los investigadores positivistas (fuertes y débiles; nodos de color amarillo y calipso, respectivamente) y los interpretativos (nodos azules) hay conexiones, aunque pocas de modo directo. La mayor parte ocurre a través de la mediación de los investigadores híbridos (rojos), que se sitúan conspicuamente en la zona central de la red. Este rol interconector queda claramente a la vista si extraemos de la red los nodos híbridos. Sin los híbridos, el centro de la red queda ocupado por los investigadores positivistas y los interpretativos quedan predominantemente conectados entre sí o aislados, y en la periferia. Tan sólo algunos investigadores interpretativos, como Kathya Araujo y Ximena Valdés, mantienen cierta centralidad y quedan bien interrelacionados con los investigadores positivistas centrales.

En otro estudio, hemos constatado el predominio del positivismo en los investigadores de la ciencia política (Ramos \& Canales, 2009); este predominio se ve aquí reflejado en las redes de esta disciplina (ver Gráfico 4). En la zona central de la red de ciencia política predominan los investigadores positivistas. Posiciones centrales son ocupadas por investigadores enmarcados en un positivismo fuerte, como Carlos Huneeus y David Altmann, mientras que los investigadores interpretativos se encuentran relegados a la periferia. Tan sólo uno de estos últimos ocupa una posición manifiestamente central: Norbert Lechner. Hay también algunos investigadores híbridos en la zona central, pero sin asumir la relevancia intermediadora que tienen en sociología; su carácter híbrido además es menos marcado. 


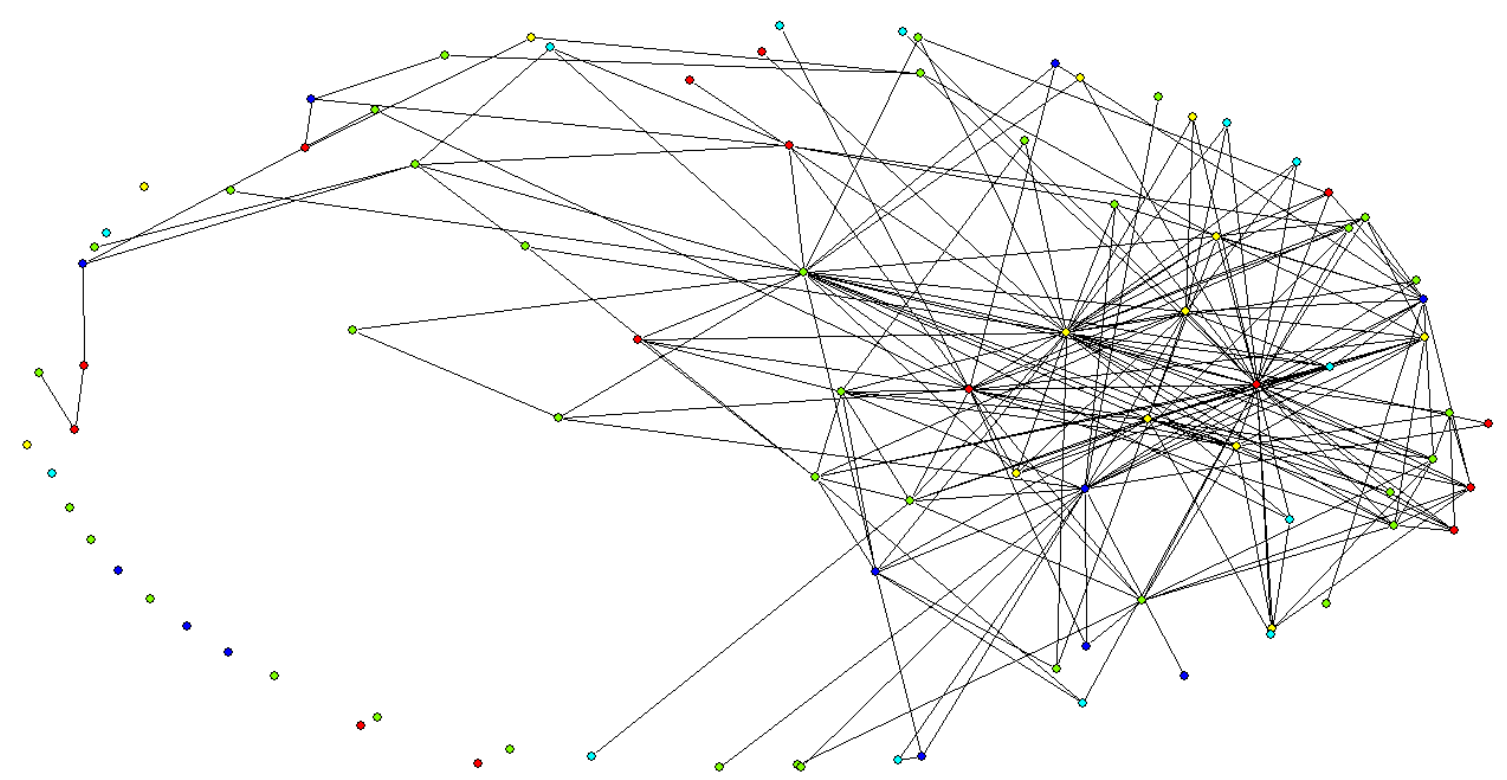

Gráfico 4: Red de comunicaciones de la ciencia política considerando encuadres paradigmáticos.

En la red de antropología social la situación es la inversa a la que se constata en la ciencia política (ver Gráfico 5); los investigadores que se encuadran en el paradigma interpretativo son los que ocupan las posiciones centrales y están más interconectados entre sí. ${ }^{11}$ Los investigadores positivistas, por su parte, están dispersos en la red, en posiciones periféricas, con muy bajo nivel de conectividad. Algunos investigadores híbridos cumplen funciones de intermediación, pero ella en general no es de carácter interparadigmática. Los nodos híbridos (rojos) están muy lejos de desempeñar el destacado papel de mediación que tienen en la red de sociología.

\footnotetext{
${ }^{11}$ Esto también concuerda con lo que hemos encontrado en el análisis del contenido de los textos del corpus de antropología (ver Palestini, Ramos \& Canales, 2010).
} 


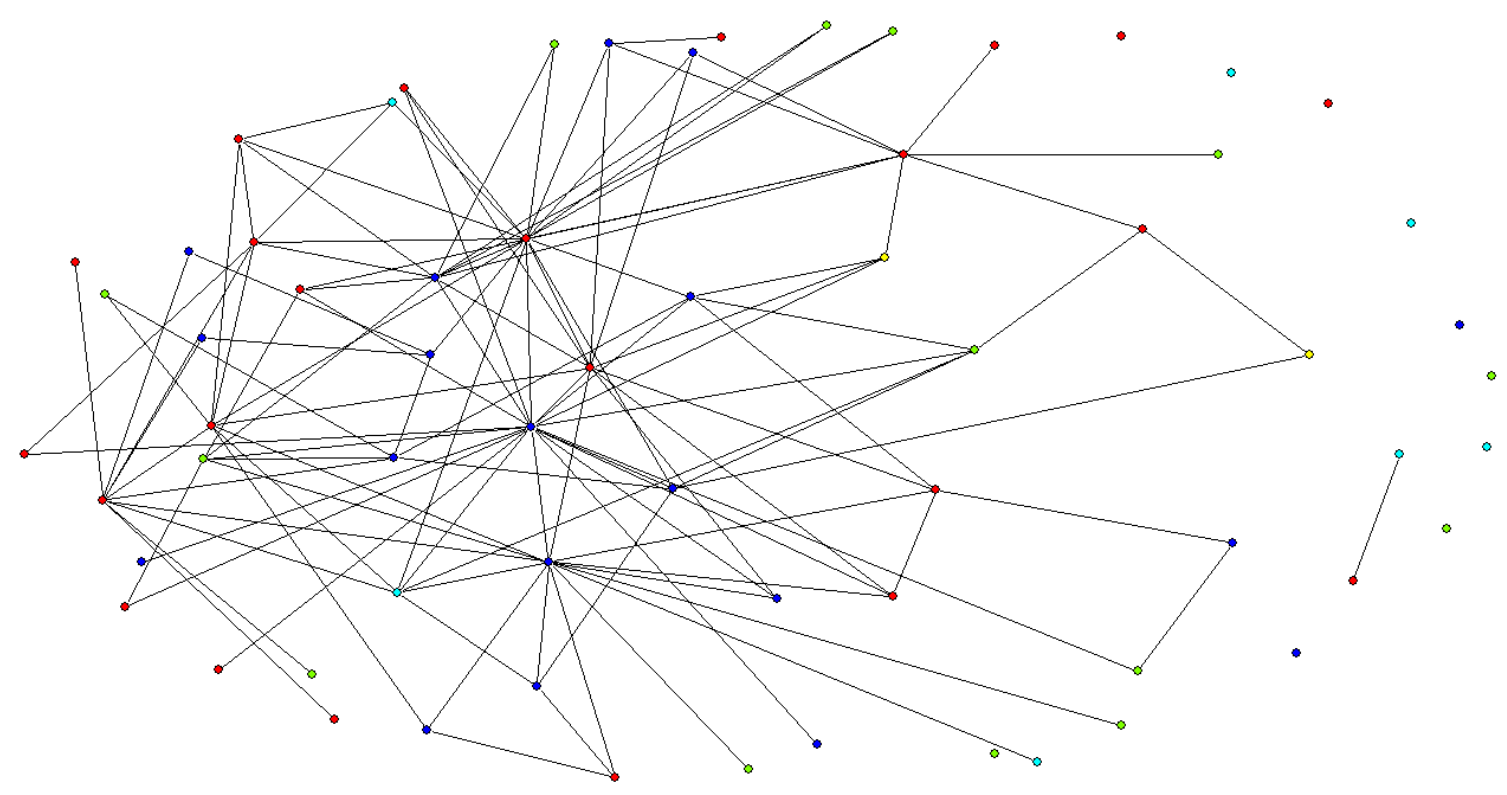

Gráfico 5: Red de comunicaciones de la antropología considerando paradigmas científicos.

\section{Redes e instituciones}

La incidencia del factor institucional en las agrupaciones de redes lo veremos en términos agregados, en cuanto a los tipos de institución en que se produce el conocimiento científico social. En el gráfico siguiente nuevamente tenemos la red global, coloreada ahora de acuerdo al tipo de institución en la cual el investigador realiza fundamentalmente su trabajo: universidades complejas, tradicionales y metropolitanas, en amarillo; universidades tradicionales de regiones, en verde claro; universidades privadas, en rojo; organismos internacionales, en verde oscuro; centros académicos independientes, en azul; y organismos estatales, en calipso. Los nodos sin vínculos institucionales o para los que no se cuenta con información están en gris. 


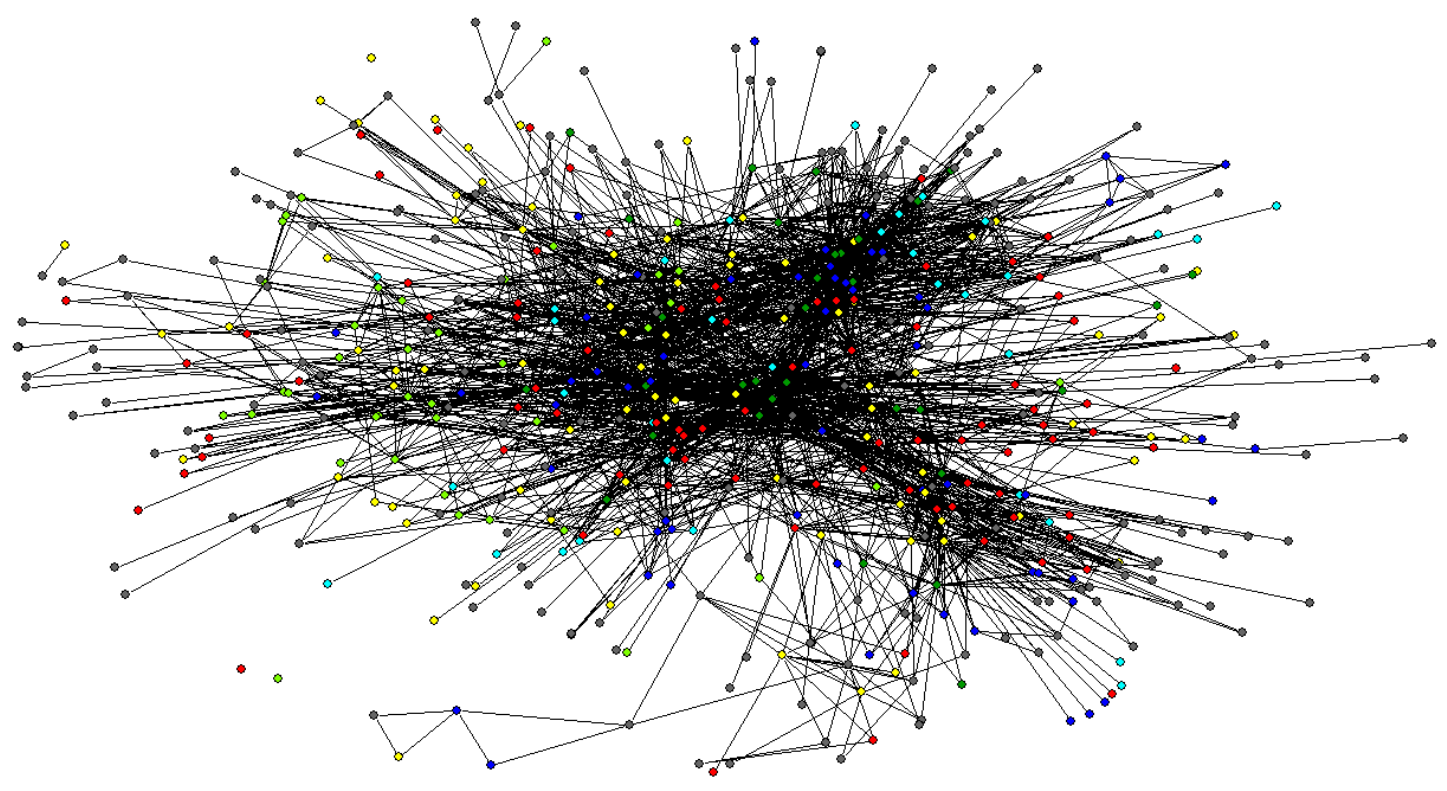

Gráfico 6: Red del campo total e instituciones de producción de conocimientos.

En este caso las demarcaciones no son tan nítidas. Las instituciones de producción científica no tienen el poder para concentrar conexiones que tienen las disciplinas y una primera constatación es que hay un gran entrelazamiento general entre investigadores de diferentes tipos de instituciones. No obstante, ello ocurre con ciertas pautas discernibles.

Si antes de 1973 las universidades ocupaban las posiciones centrales y en el período de la dictadura lo hacían los centros académicos independientes, la situación actual es más compleja. En el período presente, el centro de la red aparece compartido entre centros académicos independientes (nodos azules), universidades privadas (nodos rojos), grandes universidades tradicionales (nodos amarillos) y organismos internacionales (nodos verdes oscuros). Todas esas diversas instituciones comparten la zona central y no podría decirse que ninguna de ellas tenga el dominio de la zona. Junto con este balance, muy diferente a la situación de períodos previos, el otro hecho novedoso es el nítido posicionamiento que han alcanzado las universidades privadas (nodos rojos) en las redes científico sociales. Son un nuevo actor que ya ha alcanzado relevancia en el campo. 
Destaca además que tanto los centros independientes como las universidades privadas aparecen más interconectados entre sí que las grandes universidades tradicionales, cuyos integrantes (nodos amarillos) se encuentran más dispersos y desconectados entre ellos.

Por otra parte, tal como anticipábamos, las universidades de regiones no forman parte del tejido comunicativo central del campo. Algunos investigadores de regiones integran subredes, pero éstas no alcanzan centralidad en el campo total. Tan sólo unos pocos investigadores de regiones han logrado cierta centralidad global.

\section{Red de comunicaciones y género}

El segundo factor externo al proceso cognitivo mismo de la ciencia que hemos considerado es el género. ¿Incide el género en la configuración de las redes? ¿Pueden discernirse pautas preferenciales de conexión asociadas al género? En el Gráfico 7 aparece la red total del campo coloreada de acuerdo a género: hombres en azul, mujeres en amarillo.

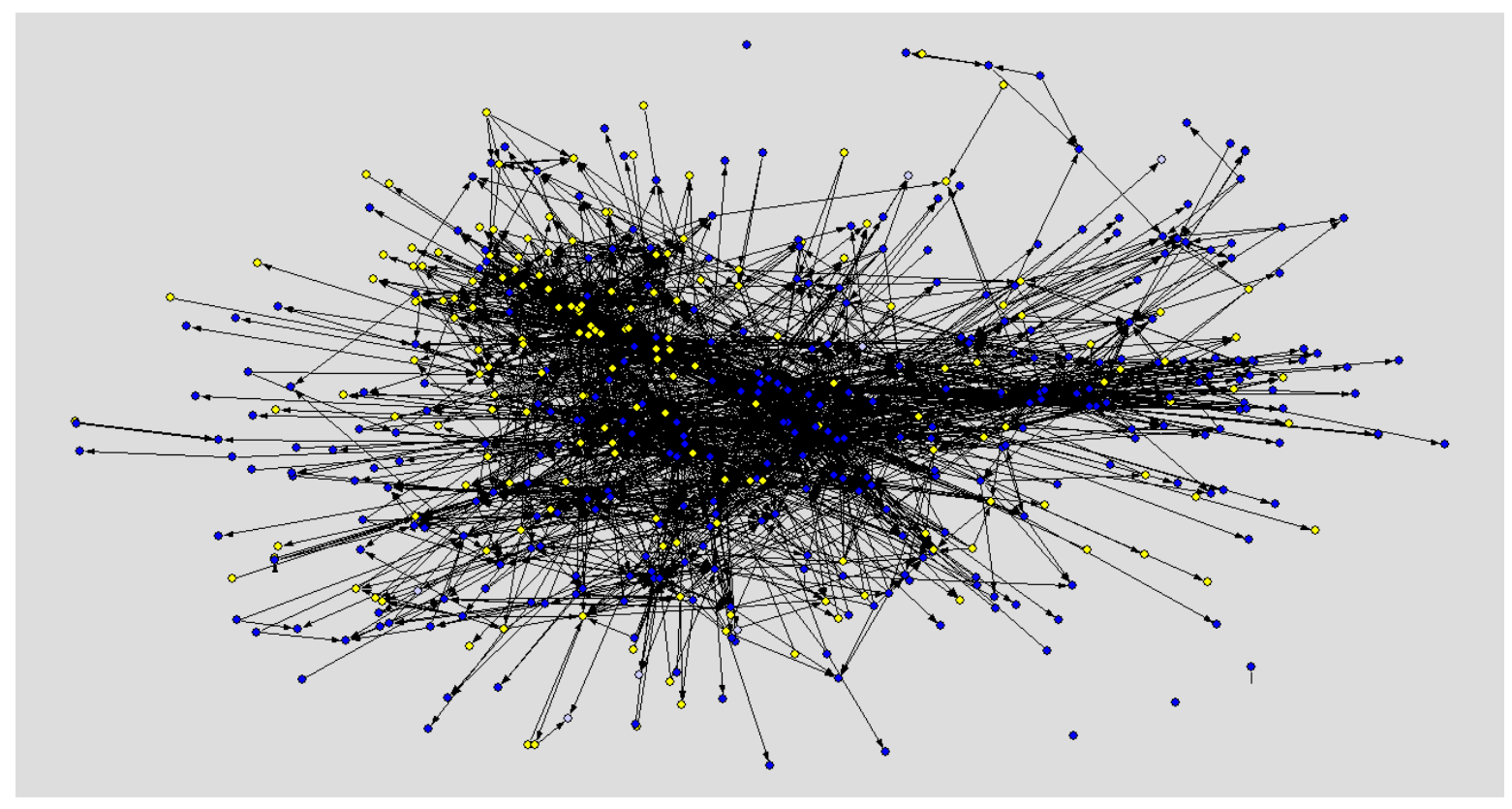

Gráfico 7: Red de comunicaciones del campo total y género.

Sorprende la marcada concentración por género que presentan las redes de referencias. En el área superior izquierda se visualiza claramente una marcada concentración de relaciones entre autoras mujeres. En contraste, hacia la derecha hay entretejimientos de relaciones en que predominan los hombres. Es una peculiar segregación por género, inesperada en su nitidez. 


\section{Redes de colaboración}

La colaboración entre investigadores del campo de las ciencias sociales en Chile, expresada en coautorías, ocurre en el $40.1 \%$ de los textos, cifra que está cercana a los niveles internacionales (Gingras, 2002; Larivière et al., 2006) y es especialmente alta en sociología (49.3\%), donde también se encuentran los equipos más grandes de coautoría (ver Tabla 4), lo cual podría expresar la mayor complejidad de los proyectos de investigación en que se embarca esta disciplina en comparación a las otras.

\begin{tabular}{|c|c|c|c|c|}
\hline \multirow{2}{*}{$\begin{array}{c}\text { CANTIDAD DE } \\
\text { AUTORES }\end{array}$} & TOTAL & Sociología & Ciencia Política & Antropología \\
\cline { 3 - 5 } & 59.9 & 50.7 & 65.5 & 66.0 \\
\hline 1 & 24.6 & 27.2 & 23.0 & 20.8 \\
\hline 2 & 9.4 & 13.4 & 6.7 & 8.5 \\
\hline 3 & 3.8 & 4.9 & 3.6 & 4.7 \\
\hline 4 & 2.3 & 3.8 & 1.2 & 0 \\
\hline 5 y más & $\begin{array}{c}100.0 \\
(479)\end{array}$ & $\begin{array}{c}100.0 \\
(268)\end{array}$ & $\begin{array}{c}100.0 \\
(165)\end{array}$ & $\begin{array}{c}100.0 \\
(106)\end{array}$ \\
\hline
\end{tabular}

Tabla 4: Cantidad de autores en los textos del corpus, según disciplina (en \%).

De los investigadores presentes en el corpus de textos, 312 participan en estas relaciones de colaboración. Esta es una red muy disgregada, con un coeficiente de fragmentación de 0.97 (Borgatti, 2003) y muy baja densidad (0.009), lo cual se puede apreciar gráficamente en la imagen siguiente conteniendo la red (Gráfico 9). 


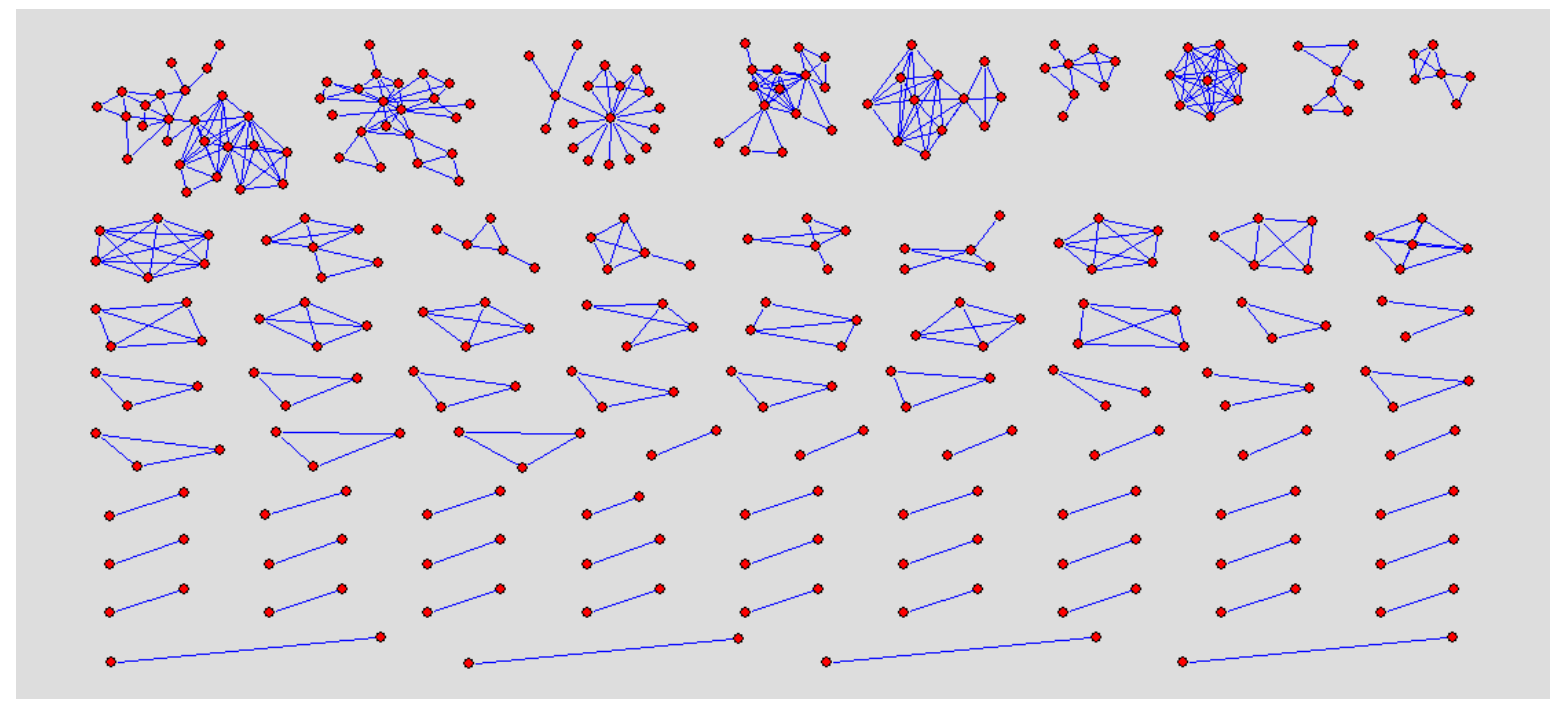

Gráfico 9: Red de colaboración en el campo total.

En total, en el campo hay 76 componentes, es decir agrupaciones de investigadores conectados entre sí por este tipo de colaboración, y que no están conectados con otros. Naturalmente, esta red de colaboración, como estructura total, tiene un grado de jerarquía de Krackhardt muy bajo y lo mismo ocurre con las medidas de centralización.

A su vez, la colaboración internacional, tal como preveíamos, es baja. Tan sólo el $2.1 \%$ de los textos incluyen la participación de uno o más autores extranjeros, lo cual muestra un rezago en Chile con respecto a esta tendencia global.

Dentro de esta red descentralizada hay varios clusters interesantes, que además representan focos significativos de productividad. Varias de las mayores agrupaciones de colaboración tienen una composición predominantemente femenina. De hecho, los 7 investigadores conectados a un mayor número de cliques -es decir, a grupos de investigadores conectados entre sí- son todos mujeres, conectadas a entre 3 y 5 cliques distintas. ${ }^{12}$ Esto señala la relevancia de las mujeres en el establecimiento de colaboraciones investigativas en el país. Por otra parte, se observa que la colaboración establecida por las mujeres es fundamentalmente con otras mujeres, reiterando la pauta de conexión por género que habíamos anotado antes y además presenta una marcada horizontalidad en las relaciones.

12 Técnicamente, una clique es un subgrafo completo y máximo; es decir, un subconjunto de nodos en el cual todo par posible de nodos está directamente conectado y este grupo no está contenido en otro (Scott, 2000: 114). 


\section{Conclusiones}

Los resultados muestran un campo de las ciencias sociales en Chile con baja densidad y fragmentado, con varias líneas de fractura, algunas de carácter propiamente epistémico, otras de carácter institucional o sociocultural. Las disciplinas, tal como planteábamos hipotéticamente, ponen barreras a la colaboración, siendo especialmente marcadas las fronteras entre la ciencia política y la antropología, casi carentes de comunicaciones entre sí. El análisis de redes permite ver el rol especial que juega la sociología, operando como sub-red interconectora, mediadora entre las otras disciplinas. Sin la sociología, el campo de las ciencias sociales, como tal, desaparecería, se anularía la red general. ¿Qué ha hecho que la sociología llegue a desempeñar tal rol? Podemos bosquejar algunos intentos de respuesta. Su mayor diversidad temática y conceptual contribuye seguramente a que juegue ese rol conector; de hecho, de las tres disciplinas es la que tiene una mayor diversidad semántica (Ramos et al., 2008), la cual probablemente ha sido favorecida por su más prolongado proceso de institucionalización. También cabe mencionar que en el desarrollo histórico de las ciencias sociales en Chile la sociología ocupó un lugar central. Expresión de ello es su papel en la Facultad Latinoamerica de Ciencias Sociales (Flacso), una de las más importantes entidades formadoras de investigadores sociales en Chile (Franco, 2008). Por otra parte, son las teorías de la sociología las de más amplio uso en el campo. Todo esto incididiría en la centralidad de la sociología que se constata en las redes.

Los encuadres paradigmáticos, que representan tradiciones y culturas epistémicas diferentes, también constituyen focos de conglomeración dentro de cada uno y de separación con los otros encuadres. Esto ocurre con mayor intensidad en la antropología y ciencia política; en la sociología, en contraste, los paradigmas, aunque siguen operando como focos de concentración de conexiones, exhiben una mayor permeabilidad. En ello incide de manera destacada el rol de brokers jugado por un conjunto de investigadores quienes en su trabajo de generación de conocimientos presentan hibridez paradigmática, mezclando componentes paradigmáticos u oscilando entre un marco paradigmático y otro. Estos investigadores ocupan posiciones centrales -tienen alta centralidad in degree así como de intermediación- y facilitan el acercamiento interparadigmático. 
Las redes del campo reflejan una nueva articulación de la institucionalidad científico social en el país. Ha emergido con fuerza un nuevo actor institucional: las universidades privadas, las cuales muestran un dinamismo y una cantidad de interconexiones parecidos al de los centros académicos independientes; estos últimos siguen siendo centrales, aunque ya no tienen el predominio que alcanzaron bajo la dictadura militar de Pinochet. Las universidades tradicionales, por su parte, han recuperado su relevancia, pero sus integrantes se encuentran comparativamente más dispersos. Además, la tradición centralista sigue imponiéndose y los centros regionales mantienen posiciones periféricas en las comunicaciones del campo. Las instituciones de producción de conocimientos no aparecen, en todo caso, como un factor poderoso de fragmentación del campo (salvo en cuanto a la diferenciación regional); no tienen el marcado grado de efecto que tienen las divisiones disciplinares y paradigmáticas.

En este campo poco denso y con separaciones disciplinares y epistémicas, los investigadores apelan en exceso a la auto-cita, probablemente como estrategia apropiada para la difusión y visibilización de la propia producción y, por otra parte, existe una alta tasa de investigadores no citados, de conocimientos no aprovechados, de conocimientos no enlazados. ${ }^{13}$ La proporción resulta más alta que la habitual internacionalmente (De Solla Price, 2006) y revela problemas en el funcionamiento del campo. Es sintomático de esto que los investigadores activos hagan un uso relativamente reducido de los conocimientos que sus colegas están produciendo en el mismo período. En esto muy probablemente inciden la mencionada fragmentación y también las debilidades en el sistema de difusión de conocimientos dentro del campo.

La investigación reveló, además, un significativo e inesperado rol del género en la constitución de las redes. Por una parte, el campo presenta claros clivajes marcados por el género. Por otra parte, las mujeres presentan una mayor propensión colaborativa que los hombres, la cual se expresa en redes de colaboración más extensas y de carácter horizontal; tal carácter horizontal contrasta fuertemente, además, con la verticalidad o centralización de algunas redes de predominio masculino.

\footnotetext{
${ }^{13}$ En las diferentes redes disciplinares hay alrededor de un cuarto de todos los investigadores que, en el período de los siete años analizados, no han recibido ninguna cita por otros investigadores en referencia a sus trabajos, lo cual refleja un importante grado de pérdida del producto investigativo.
} 


\section{Bibliografia}

Bourdieu, Pierre (1988). Homo Academicus. Stanford, Calif.: Stanford University Press. Bourdieu, Pierre (1999). "El campo científico". En Pierre Bourdieu, Intelectuales, política y poder. Buenos Aires: Eudeba.

Bourdieu, Pierre (2001). Science de la science et réflexivité. Paris: Raisons d'Agir Editions.

Callon, Michel \& John Law (1998). "De los intereses y su transformación. Enrolamiento y contraenrolamiento". En Miquel Doménech, Francisco Tirado, comps. Sociología simétrica. Ensayos sobre ciencia, tecnología y sociedad. Barcelona: Gedisa.

Callon, Michel; Jean-Pierre Courtial; William Turner y Serge Vauin (1983). "From translations to problematic networks: An introduction to co-word analysis". Social Science Information, 22(2): 191-325.

Carley, Kathleen (2002). Summary of Key Network Measures for Characterizing Organizational Architectures. Unpublished document. Carnegie Mellon University.

Clemens, Elisabeth; Walter Powell; Kris McIlwaine, y Dina Okamoto (1995). "Careers in Print: Books, Journals, and Scholarly Reputations". American Journal of Sociology, 101(2): 433-494.

De Bellis, Nicola (2009). Bibliometrics and Citation Analysis. Lanham, Maryland: The Scarecrow Press.

De Haan, J. (1997). "Autorship Patterns in Dutch Sociology". Scientometrics, 39(2): 197-208.

De Nooy, Wouter; Andrej Mrvar y Vladimir Batajelj (2005). Exploratory Social Network Analysis with Pajek. Cambridge: Cambridge University Press.

Degenne, Alain \& Michel Forsé (2004). Les réseaux sociaux. Paris : Armand Colin.

De Solla Price, Derek (2006). "Networks of Scientific Papers". En Newman, Mark; Albert-László Barabási y Duncan Watts (eds.) The Structure and Dynamics of Networks. Princeton: Princeton University Press.

Fourcade, Marion (2009). Economists and Societies. Discipline and Profession in the United States, Britain \& France, 1890s to 1990s. Princeton: Princeton University Press.

Franco, Rolando (2008). La FLACSO clásica. Santiago, Chile: FLACSO / Editorial Catalonia. 
Garretón, Manuel Antonio (2005). "Social Sciences and Society in Chile: Institutionalization, Breakdown and Rebirth". Social Science Information, 44 (2 \&3): 359-409.

Gilbert, Nigel (1977). "Referencing as Persuasion". Social Studies of Science, 7(1): 113-122.

Gingras, Ives (2002). "Les formes spécifiques de l'internationalité du champ scientifique". Actes de la Recherche en Sciences Sociales, 141(2): 31-45.

Gmür, Markus (2003). "Co-citation Analysis and the Search for Invisible Colleges: A Methodological Evaluation". Scientometrics, 57(1): 27-57.

Hargens, Lowell (2000). "Using the Literature: Reference Networks, Reference Contexts, and the Social Structure of Scholarship". American Sociological Review, 65(6): 846-865.

Hicks, Diana (1987). "Limitations of Co-Citation Analysis as a Tool for Science Policy". Social Studies of Science, 17: 295-316.

Knorr-Cetina, Karin (2005). La fabricación del conocimiento: Un ensayo sobre el carácter constructivista y contextual de la ciencia. Buenos Aires: Universidad Nacional de Quilmes Editorial.

Krackhardt, David (1994). Graph Theoretical Dimensions of Informal Organizations. En Carley, Kathleen y M.J. Prietula (eds.) Computational Organization Theory. Hillsdale, NJ: Lawrence Erlbaum Associates.

Kuhn, Thomas (1971). La estructura de las revoluciones científicas. México: Fondo de Cultura Económica.

Larivière, Vincent; Yves Gingras y Eric Archambaul (2006). "Canadian Collaboration Networks: A Comparative Analysis of the Natural Sciences, Social Sciences and the Humanities". Scientometrics, 68(3): 519-533.

Latora, V. \& M. Marchiori (2001). "Efficiente Behavior of Small-World Networks. Physics Review Letter, 87 (19).

Latour, Bruno, Steve Woolgar, 1986. Laboratory Life: The Construction of Scientific Facts. 2nd. ed. Princeton: Princeton University Press.

Leydesdorff, Loet (2001). The Challenge of Scientometrics. The Development, Measurement, and Self-Organization of Scientific Communications. La Vergne, TN: Universal Publishers. 
Leydesdorff, Loet (2007). "Scientific Communication and Cognitive Codification: Social Systems Theory and the Sociology of Scientific Knowledge". European Journal of Social Theory, 10(3): 375-388.

Leydesdorff, Loet \& Olga Amsterdamska(1990). "Dimensions of Citation Analysis". Science, Technology \& Human Values, 15(3): 305-335.

Leydesdorff, Loet \& P. Wouters (1999) "Between Texts and Contexts: Advances in Theories of Citation? (A Rejoinder). Scientometrics, 44(2): 169-182.

Merton, Robert K. (1973). The Sociology of Science: Theoretical and Empirical Investigations. Chicago: University of Chicago Press.

Mouton, Johann (2010). "The Internationalization of South African Social Science". En Kuhn, Michael \& Doris Weidemann (eds.), Internationalization of the Social Sciences. Bielefeld: Transcript Verlag.

Mutschke, Peter \& Anabel Quan Haase (2001). "Collaboration and Cognitive Structures in Social Science Research Fields. Towards Socio-cognitive Analysis in Information Systems". Scientometrics, 52(3): 487-502.

Nelson, Michael (2006). "Visualization of Citation Patterns of Some Canadian Journals". Scientometrics, 67(2): 279-289.

Newman, Mark (2010). Networks. Oxford: Oxford University Press.

Newman, Mark (2006). "The Structure of Scientific Collaboration Networks". En Newman, Mark; Albert-László Barabási y Duncan Watts (eds.) The Structure and Dynamics of Networks. Princeton: Princeton University Press.

Newman, Mark; Albert-László Barabási y Duncan Watts, eds. (2006). The Structure and Dynamics of Networks. Princeton: Princeton University Press.

Palestini, Stefano; Claudio Ramos y Andrea Canales (2010). "La producción de conocimiento antropológico social en el Chile post-transición: Discontinuidades del pasado y debilidades del presente". Estudios Atacameños, 39.

Porter, Alan (1977). "Citation Analysis: Queries and Caveats". Social Studies of Science, 7(2): 257-267.

Powell, Walter; Douglas White; Kenneth Koput; Jason Owen-Smith (2005). "Network Dynamics and Field Evolution: The Growth of Interorganizational Collaboration in the Life Sciences". American Journal of Sociology, 110(4): 1132-1205. 
Ramos, Claudio (2005). "Cómo investigan los sociólogos chilenos en los albores del siglo XXI: Paradigmas y herramientas del oficio". Persona y Sociedad, 19 (3): 85-119. Ramos, Claudio (2008). "Destino buscado del conocimiento científico social, forma de construcción investigativa y configuración del campo". Ponencia presentada en Congreso PreAlas Chile 2008. 50 años de sociología.

Ramos, Claudio (2012). El ensamblaje de ciencia social y sociedad. Conocimiento científico, gobierno de las conductas y producción de lo social. Santiago, Chile: Ediciones Universidad Alberto Hurtado.

Ramos, Claudio; Andrea Canales y Stefano Palestini (2008). "El campo de las ciencias sociales en Chile: ¿Convergencia disciplinar en la construcción del objeto de estudio?". Cinta de Moebio, 33(3): 171-194.

Ramos, Claudio \& Andrea Canales (2009). "Political Science in Chile: Positivistic Hegemony, Institutional Power, and Discipline Isolation". Ponencia en XXI IPSA World Congress of Political Science.

Rivellini, Giulia; Ester Rizzi y Susanna Zaccarin (2006). "The Science Network in Italian Population Research: An Analysis according to the Social Network Perspective". Scientometrics, 67(3): 407-418.

Scott, John (2000). Social Network Analysis. A Handbook. London: Sage Publications.

Wagner, Caroline \& Loet Leydesdorff (2004). "Network Structure, Self-Organization and the Growth of International Collaboration in Science". Inédito.

Wallerstein, Immanuel (1996). Open the Social Sciences. Report of the Gulbenkian Commission on the Restructuring of the Social Sciences. Stanford, California: Stanford University Press.

Wassermann, Stanley \& Katherine Faust (1994). Social Network Analysis. Methods and Applications. Cambridge: Cambridge University Press.

Watts, Duncan \& S. H. Strogatz (2006). Collective Dynamics of 'Small-World Networks". En Newman, Mark; Albert-László Barabási y Duncan Watts (eds.) The Structure and Dynamics of Networks. Princeton: Princeton University Press. 